Nouvelles perspectives en sciences sociales

\title{
Théorie de l'action et parcours de vie
}

\section{Pierre Bouchard}

Volume 1, numéro 2, mars 2006

URI : https://id.erudit.org/iderudit/602471ar

DOI : https://doi.org/10.7202/602471ar

Aller au sommaire du numéro

Éditeur(s)

Prise de parole

ISSN

1712-8307 (imprimé)

1918-7475 (numérique)

Découvrir la revue

Citer cet article

Bouchard, P. (2006). Théorie de l'action et parcours de vie. Nouvelles

perspectives en sciences sociales, 1(2), 67-114. https://doi.org/10.7202/602471ar d'utilisation que vous pouvez consulter en ligne.

https://apropos.erudit.org/fr/usagers/politique-dutilisation/ 


\title{
Théorie de l'action et parcours de vie
}

\author{
PierRe Bouchard \\ Université de Hearst \\ Ontario, Canada
}

\section{Introduction}

Depuis toujours, des individus cherchent à comprendre et à expliquer tout ce qui se passe autour d'eux. Longtemps, les questions auxquelles ne pouvaient pas répondre les sciences ont été du domaine des mythes et des croyances religieuses. La modernité, avec un développement important dans les sciences, cherche à rationaliser tous les secrets de l'univers. Ces sciences exigent une vérité relative qui repose sur la logique et sur des données empiriques. Dans cet esprit, les chercheurs des sciences sociales essaient de rationaliser tous les phénomènes sociaux'.

On peut s'interroger à savoir où en est l'évolution des sciences sociales en ce qui concerne les mystères de la pensée et de l'action humaines. Dans certaines sciences, que l'on qualifie d'« exactes», les chercheurs arrivent à établir des lois avec une certaine précision. Dans les sciences sociales, l'objet est souvent identifiable à l'humain, un être qui, par sa pensée, peut intervenir sur lui-même et sur son histoire. La

${ }^{1}$ Guy Rocher, Introduction à la sociologie générale, Montréal, Éditions Hurtubise HMH Ltée, $3^{c}$ édition, 1992. 
spécificité de sa pensée fait de lui un objet de recherche fort complexe quand ce ne serait que parce qu'il agit sur son histoire en même temps que son histoire agit sur lui-même ${ }^{2}$. Néanmoins, les chercheurs en sciences sociales tentent de produire, comme leurs collègues des sciences « exactes ", des explications qui se veulent les plus rationalistes possible. Pour y arriver, ils fondent la majorité de leurs travaux sur le paradigme de l'utilitarisme, c'est-à-dire sur l'hypothèse d'un acteur rationnel, guidé par l'intention, par la stratégie, par la conscience, par l'intérêt. Cela vaut pour la presque totalité des approches où l'individu est au centre de l'analyse : interactionnisme méthodologique, ethnométhodologie, individualisme méthodologique, théorie des choix rationnels, et même structuralisme génétique et actionnalisme. On pourrait très certainement apporter des nuances entre toutes ces approches, et elles seraient toutes intéressantes autant que justifiées ; mais les distinctions ne seront jamais telles qu'on ne trouvera pas en l'une d'elles un acteur rationnel-intentionnel, motivé par l'intérêt. Ce courant de pensée repose sur le postulat selon lequel l'action part toujours de l'intérêt et elle a pour but de maximiser les gains. De ce postulat découle une action humaine qui est comprise comme rationnelle. Un modèle utilitariste comme celui-ci apparaît suffisamment important pour qu'on le trouve au fondement de l'usage d'un grand nombre de concepts parmi les plus importants ${ }^{3}$ et au fondement de théories dans les sciences sociales ${ }^{4}$. Mais on peut se demander, comme Caillé,

Dans quelle mesure, [...] [1]]accès à cette scientificité qu'elles [les sciences sociales] proclament si fort rechercher implique-t-il qu'elles fassent l'hypothèse que l'action humaine et sociale obéit, au moins pour sa part la plus significative, à une causalité rationnelle $?^{5}$

Et, effectivement, cette notion de rationalité de l'acteur pose problème. Elle alimente des débats théoriques importants dans les sciences sociales. De récents écrits cherchent à élargir la conception de la

${ }^{2}$ Edgar Morin, I a méthode, tome 5, L'bumanité de l'bumanité, L'identité bumaine, Paris, Seuil, 2001.

${ }^{3}$ Intentionnalité, stratégies, autonomie, conscience.

${ }^{4}$ Ethnométhodologie, interactionnisme symbolique, individualisme méthodologique, ethnographie institutionnelle, théorie des choix rationnels...

5 Alain Caillé, La démission des clercs, Paris, La Découverte, 1993, p. 109. 
rationalité pour qu'elle puisse continuer à être au fondement de l'explication de l'action; on pense à la théorie des choix rationnels ou à des développements de la phénoménologie ou, plus précisément, de l'individualisme méthodologique.

La théorie des choix rationnels s'insère dans le paradigme utilitariste. Les sciences économiques, par exemple, pour une bonne part fondatrices des sciences sociales actuelles, utilisent ce paradigme pour expliquer la totalité des actions humaines. Cette perspective est celle d'un acteur libre, intentionné et motivé par ses intérêts. Dans les plus poussées de ces théories, ainsi que dans celles qui sont spécifiquement économicistes, on y perçoit même l'acteur comme étant uniquement calculateur et égoïste, ne cherchant qu'à maximiser ses gains. Cette vision permet, en fait, de faire l'économie de concepts dans l'analyse des actions humaines. C'est une modélisation qui donne l'illusion d'une imitation des sciences « exactes », illusion, soit dit en passant, qui a pour fondement la mécompréhension du travail réel qu'on effectue dans ces sciences. Toutefois, pour une majorité de chercheurs, cette forme de rationalité, c'est-à-dire utilitaire, pose des problèmes parce qu'elle laisse échapper trop de dimensions de l'action humaine.

C'est avec le besoin de ramener un acteur au centre des analyses que ce paradigme s'est imposé à la sociologie, pour ainsi contrer les modèles structuralistes qui furent dominants, modèles où l'acteur apparaît comme essentiellement déterminé en extérioritét. Néanmoins, dans la communauté scientifique, on semble de plus en plus reconnaitre les limites des théories rationalisantes. L'école rationaliste admet que certains comportements ne sont pas rationnels. Le problème, c'est qu'elle ne sait pas quoi en faire. Elle conçoit que l'être humain est nécessairement rationnel et émotionnel. Elle reconnait aussi l'importance du vécu, du contexte dans lequel a lieu l'action, et de l'historicité. Elle constate que l'être humain est toujours en relation. Pourtant, elle s'accroche à la notion de rationalité et l'institue en paradigme dominant. Or, on peut se demander pourquoi les sciences sociales hésitent à délaisser la notion de rationalité. Car nous croyons qu'il est possible pour elles d'apporter une explication rationnelle, scientifique, sans pour autant prétendre à la pure rationalité de l'acteur.

"Simon Laflamme, Communication et émotion. Essai de microsociologie relationnelle, Paris, L'Harmattan, 1995. 
On trouve diverses critiques du paradigme utilitariste. Il y a celle de la socioéconomie (Society for Advancement for Socio-Economics (SASE)) qui est née aux États-Unis. Il y a celle de la sociologie économique dont Granovetter et Swedberg font figure de chefs de file. Il y a aussi le Mouvement antiutilitariste en sciences sociales (MAUSS) dont les principaux représentants sont Caillé et Godbout. Ces trois courants optent pour la multidisciplinarité. Tous les trois expriment une insatisfaction à l'égard de l'économie néoclassique. Tous les trois sont à la recherche de théories de remplacement. Mais c'est manifestement le MAUSS qui pose le plus explicitement la question de la légitimité du paradigme utilitariste.

Dans la pratique, cependant, les chercheurs semblent peu s'intéresser au débat entourant la rationalité de l'acteur. Dans l'ensemble, les praticiens adoptent une approche phénoménologique où l'analyse s'effectue à partir d'un sujet intentionné, calculateur, autonome et intéressé. L'objectif de cette recherche dans laquelle nous nous engageons est de montrer les problèmes qui sont liés à l'utilisation, dans les théories des sciences sociales, de la notion de rationalité de l'acteur. Son hypothèse générale veut que ces sciences font un usage abusif de la notion de rationalité dans l'explication de l'action humaine.

Comment expliquer l'importance de la pensée utilitariste dans les sciences sociales? On peut recourir à deux hypothèses :

- Les sciences sociales ont besoin d'une logique qui permette de produire des concepts, des théories, des modèles qui peuvent répondre à certains critères du mode de pensée scientifique. Les sciences économiques ont développé, dans cet esprit, une logique utilitariste réductrice.

- L'utilitarisme permet de produire des modèles qui remettent l'acteur au centre de l'analyse. Pour les défenseurs de l'acteur, il était important de s'opposer au courant structuraliste qui réduisait l'acteur à un être passif, déterminé par les structures.

À ces deux hypothèses, on peut ajouter qu'il faut en voir la raison dans le fait : 1) qu'il simplifie l'analyse de l'action et 2) qu'on ne trouve pas vraiment de modèles de remplacement. ${ }^{7}$ 
Au point de départ, cette recherche entend prouver que les modèles d'analyse de l'action qui postulent le caractère essentiellement rationnel de l'action sociale abusent du concept. Ainsi, pour éliminer les lacunes de la théorie des choix rationnels, tout en conservant un acteur libre et autonome, les sciences sociales élargissent la définition du concept de rationalité. Cette manière de faire s'insère dans le débat acteur/structure où l'on ne parvient pas à concevoir une action «émorationelle », où «les acteurs socialisés agissent sur les structures qui les déterminent $»^{8}$. C’est l'ensemble de cette problématique que doivent prendre en considération les modèles de remplacement et ils ne peuvent le faire s'ils postulent que l'action est strictement rationnelle?.

L'analyse des données auxquelles a pu accéder cette étude révèle des dimensions négligées par ce modèle. Il semble que, dans une logique utilitariste, l'émotion, la socialité, les structures, soient délaissées pour ne retenir que la rationalité comme élément explicatif de l'action. Même si certains ${ }^{10}$, par acquis de conscience, mais sans vraiment altérer le modèle utilitariste, tentent de tenir compte du vécu et des structures, peu s'intéressent à l'émotion, pourtant un élément inhérent à l'action.

\section{Acteur : rationnel et émotionnel}

L'ensemble des théories utilitaristes de même que la plupart des théories antiutilitaristes ne conçoivent pas l'acteur comme essentiellement social. Dans cet esprit, les modèles d'analyse cherchent à singulariser l'acteur. L'erreur vient du fait que l'acteur est toujours en relation, il n'existe que dans le relationnel. La relationalité caractérise de façon essentielle l'acteur social de telle manière que l'idéal d'autonomie que définit l'utilitarisme n'est au mieux que caricatural puisque l'acteur n'existe qu'en relation et que les dynamiques des dispositifs dans cette relation ne peuvent que limiter les possibilités d'action. À partir de ces constats, on peut résumer ainsi les trois problèmes fondamentaux que rencontre la rationalité utilitaire:

\footnotetext{
${ }^{8}$ Simon Iaflamme et Rachid Bagaoui, « Don, raison et émotion », Revue de l'Institut de sociologie, vol. 2003, à paraître.

${ }^{9}$ Taché, Alain, L'adaptation, un concept sociologique systemique, Paris, L'Harmattan, 2004.

${ }^{11}$ Michel Crozier et Erhard Friedberg, par exemple.
} 
- la complexité des mondes fait que l'acteur ne peut appréhender que de façon diffuse l'ensemble d'une situation ;

- le caractère indissociable de l'émotion et de la rationalité dans l'action fait en sorte que l'analyse utilitariste tronque toujours à quelque degré son objet ;

- l'action est essentiellement relationnelle.

\section{Modélisation de la compréhension de l'action dans une perspective relationnelle}

Le paradigme utilitaire, d'une part, abuse de certains concepts et, d'autre part, semble délaisser des éléments importants de l'action dans ses modèles d'analyse. L'appareil conceptuel comprend deux perspective : une perspective utilitariste, qui présente les concepts de rationalité, de conscience, d'intérêt, de stratégie, d'autonomie, d'intentionnalité et de calcul; et une perspective relationnelle, qui présente les concepts d'émorationalité, d'acteur/structure, de relation et de complexité.

On note que, dans la perspective utilitariste, l'appareil présente des concepts que cherche à légitimer la notion de rationalité pour expliquer l'action humaine. Cette détermination rationnelle de l'acteur s'explique par le fait que le modèle a besoin de conserver l'acteur au centre de l'analyse. À la différence de celle-ci, une perspective relationnelle étend l'ensemble des éléments constitutifs de l'action, ce qui a pour effet de l'éloigner d'un modèle centré sur l'acteur tout en complexifiant les qualités, et donc tout en s'opposant à une sursimplification de l'explication de l'action humaine.

La compréhension de l'action exige, selon nous, qu'on respecte trois postulats. Le premier postulat : concevoir l'action dans la dialectique acteur/structure, en ce que "les acteurs socialisés agissent sur les structures qui les déterminent ${ }^{11}$. Le deuxième : toute action est nécessairement émorationnelle ; toute action contient une part d'émotion et une part de rationalité. Le troisième, enfin : l'acteur est essentiellement un être relationnel; il n'existe que dans et par des relations. Ce n'est qu'en assumant ces trois postulats qu'il est possible, selon nous, de concevoir et de produire des modèles d'analyse qui renverseront les bases théoriques de l'axiomatique utilitariste.

${ }^{11}$ Simon Iaflamme et Rachid Bagaoui, op. cit. 
Le schéma 1 illustre cette nouvelle compréhension de l'action humaine. Le modèle tient compte des éléments qui sont écartés par les modèles utilitaristes, mais dont cette recherche démontrera empiriquement qu'ils sont indispensables à l'analyse de l'action humaine.

\section{Schéma 1}

Modélisation de la compréhension de l'action dans une perspective relationnelle

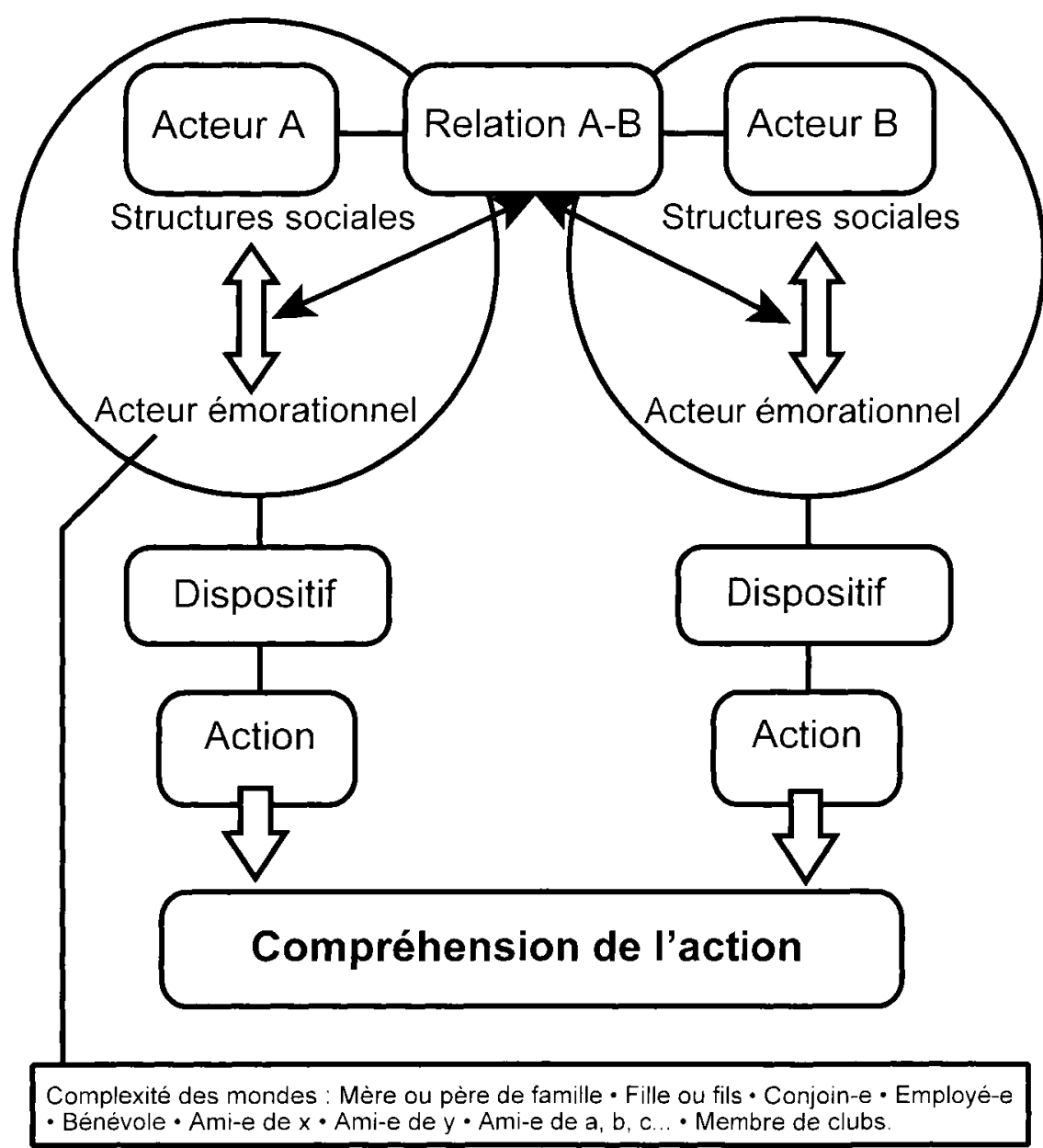




\section{Compréhension de l'action}

Ce modèle présente les dynamiques qui sont présentes dans la compréhension de l'action. Dans ce schéma, l'Acteur A et l'Acteur B sont dans une relation A-B. Dans cette relation, on trouve chez chacun des acteurs un dispositif précis qui est le produit de leur relation. En même temps, il existe une dynamique entre les structures sociales et l'acteur émorationnel. Ces dynamiques sont historicisées et se transforment continuellement.

En réduisant la part de rationalité dans l'analyse, on constate que l'acteur n'agit plus par choix, par intérêt, qu'il n'est pas un être libre et autonome comme le revendiquent les adeptes de l'action rationnelle. L'action requiert un dispositif précis. L'action dépend d'une structure. Ce dispositif appartient à la dynamique de chacune des relations et c'est par cette dynamique que se transforme le dispositif.

Dans l'ensemble, les sciences sociales reconnaissent la dialectique de l'acteur et des structures sociales. L'acteur, par tradition théorique, est du côté du vécu, de la socialisation, des valeurs, des croyances, alors que les structures sociales représentent les règles, les systèmes qui s'imposent à l'individu. Cette dialectique s'inscrit dans le temps, dans l'histoire. Elle est le moteur des transformations sociales qui circonscrivent l'action, laquelle, en retour, les produit et les transforme.

En ce sens, on ne peut pas échapper à la compréhension de l'action comme minimalement émorationnelle. Les résultats de cette recherche montreront clairement que l'ensemble des actions n'échappe pas à l'émotion. L'acteur éprouve des émotions dans chacune de ses actions : plaisir, satisfaction, amour, stimulation, déception, peur, défi, attentes, obligation ou responsabilité. En même temps, l'action est rationnelle puisque l'individu communique, ce qui suppose une rationalité, grâce à laquelle il peut intelliger son environnement et son comportement et avec laquelle il peut produire des arguments ou y accéder. Il ne pourrait y avoir de sens au plan strictement émotif. L'acteur est toujours en situation émorationelle. Toute action comporte une part d'émotion et une part de raison. Enfin, l'acteur est un être relationnel. L'acteur est toujours en relation. Il n'existe que dans la relation. Il est donc nécessaire de prendre en considération l'aspect relationnel dans la compréhension de l'action. 


\section{PIERRE BOUCHARD / THÉORIE DE L'ACTION ET PARCOURS DEVIE}

En somme, il serait bénéfique de travailler avec le principe qui veut que toute action comporte une dimension émotionnelle et rationnelle, et donc d'opérationnaliser le principe d'émorationalité. L'émotion et la raison se transforment à travers la multitude de relations qui marquent l'histoire de l'acteur. C'est la relation entre êtres humains historicisés qui doit être l'objet d'analyse. De la relation mère/enfant naît l'amour, cet amour n'est possible que par une construction historique des relations antérieures et de cette relation même. Ce faisant, on peut comprendre qu'une relation où la mère donne beaucoup d'amour à son enfant soit le résultat de ses relations historiques positives ou négatives antérieures et qu'elle procède de toutes les relations dans lesquelles la mère est agent aussi bien que de ce qui se révèle entre l'enfant et elle, et entre l'enfant et les autres personnes avec lesquelles il est en interaction. La relation vécue est nécessairement liée aux relations passées sans pour autant qu'elles en soient la source unique d'explication. ${ }^{12}$ Le vécu est le construit de toutes les relations antérieures qui interagissent et qui influencent la relation présente.

\section{Action économique vue comme émorationnelle}

Le mouvement antiutilitariste cherche à montrer que les actions ne peuvent être réduites à l'intérêt, on l'aura compris. Par contre, même les auteurs les plus critiques semblent retenir une raison utilitaire pour tenir compte de la dimension économique d'une action. Selon Caillé, " [l]e système de l'économie de marché est le seul domaine de la pratique humaine à propos duquel l'axiomatique utilitariste de l'intérêt puisse sembler immédiatement adéquate $»^{13}$. Pour sa part, Michel Bauer indique que, « [d] ans le monde des entreprises, seule la rationalité économique est reconnue comme rationalité légitime. [...] Pour être crédible et écouté, il faut obéir à la règle du 'parler-seulement-économique' ${ }^{14}$. Il

${ }^{12}$ « $[\mathrm{U}] \mathrm{n}$ individu ne s'insère pas dans une relation sans porter en lui la trace du passé. Mais ['exemple d'une relation amoureuse et celui du retour sur l'histoire respective à chacun des amants] montrent aussi que s'il faut chercher dans l'histoire les causes de ce qu'il advient, cela ne signifie pas que le devenir soit programmé par le passé " (Simon Laflamme, op. cit., p. 153).

${ }^{13}$ Alain Caillé, Critique de la raison utilitaire, Paris, La Découverte, 1989, p. 69.

${ }^{14}$ Michel Bauer, «Patron de PME, âge et rationalité économique », Gérontologie et Société, n" 70, 1994, p. 140. 
y aurait bien Michel Amiot qui, dans Les misères du patronat... ${ }^{15}$, élabore une perspective un peu différente exposant essentiellement les contraintes imposées aux patrons des PME. Par contre, en présentant deux logiques pour comprendre les actions des patrons, la logique d'entreprise et la logique d'argent, il réhabilite l'axiomatique utilitariste. La logique d'entreprise serait le désir d'œuvre, c'est-à-dire le désir de bâtir une entreprise, de créer de l'emploi, de participer au bien-être de sa communauté. La logique de l'argent se limiterait au désir d'accumuler de l'argent.

On tient souvent l'action économique pour la plus rationnelle qui soit. Dans une perspective utilitariste, la décision d'un patron est le résultat d'un calcul rationnel. Des auteurs comme Caillé, Bauer et Amiot, bien qu'ils reconnaissent quelque fondement au principe utilitariste dans le registre de l'économie, notent la difficulté liée au fait d'exclure l'émotion, même du domaine de l'économie. Mais le problème est plus grave. Tous les trois supposent que l'économique n'est qu'une des dimensions de l'action et que cette dimension pourrait être dissociée de l'ensemble des éléments qui forment l'action. Ils supposent que l'action économique est en soi une dimension distincte de l'action humaine. Or, rien n'est moins certain, tant les composantes de l'activité humaine sont imbriquées ${ }^{16}$. Entre autres, l'action économique elle-même ne peut pas se comprendre correctement si on la réduit à un simple échange effectué par un individu calculateur rationnel. C'est pourquoi,

[1]]action économique ne peut pas être considérée comme coupée de l'action sociale. Les acteurs économiques, plaide Granovetter, sont en fait toujours inscrits dans des réseaux de relations interpersonnelles, des relations proprement sociales d'alliance ou de confiance. Aucune décision économique ne peut être prise indépendamment de ces réseaux..$^{17}$

${ }^{15}$ Michel Amiot, Les misères du patronat... Le monde des petites et moyennes entreprises industrielles et de leurs patrons, Paris, L'Harmattan, 1991.

${ }^{16}$ Simon Laflamme, La société intégrée. De la circulation des biens, des idées et des personnes, New York/Paris, Peter Lang, Worcester polytechnic institute, Studies in Science, Technology and Culture, 1992.

17 Pour une autre économie, Revue semestrielle du MAUSS, $\mathrm{n}^{\circ}$ 3, Paris, Éditions La Découverte, 1994, p. 9. 
Pour Granovetter, le fait des multisituations de l'acteur oblige non seulement à prendre en considération l'action économique en elle-même mais aussi à tenir compte de l'ensemble des relations de cet acteur.

Bauer et Amiot introduisent l'aspect émotionnel dans l'action. Selon Bauer, on reconnaît l'émotion dans l'action, mais on cherche à supprimer l'émotion de l'économique pour en faire un objet plus rationnel.

Comment pourraient-ils dans un univers caractérisé par des exigences
d'efficacité et de rationalité, de modernité et de compétence, avouer ces
réalités plus «traditionnelles » qui relèvent de l'affectif et du politique,
des sentiments et de la parenté, du plaisir et du pouvoir ? [...] Tous les
discours « sérieux » font, ainsi, apparaître le chef d'entreprise comme
un être prenant toutes ses décisions selon la seule rationalité écono-
mique. Tout autre discours non économique devient sacrilège au
sommet des PME. ${ }^{18}$

Dans le même ordre d'idées,

Si la motivation principale n'était que le désir de gagner de l'argent, l'éthique patronale de la création d'entreprise se réduirait à la formule simple de l'ascétisme provisoirement accepté en vue d'une jouissance différée. Mais entre le désir de faire fortune et sa réalisation, s'interpose l'entreprise, qui incarne le désir d'œuvre, dont la réalisation, comme le plaisir qu'elle procure et le travail qu'elle exige, est immédiate, permanente, et ne peut être différée. ${ }^{19}$

On voit comment, au niveau pratique, le concept de rationalisation peut prétendre avoir sa place. Amiot sent le besoin de distinguer désir d'argent et désir d'œuvre. En fait, le désir de faire de l'argent et le désir d'accomplir une ouvre sont au même sens des émotions. Il y a autant d'émotion dans le premier désir que dans le second. Godbout, lucide, écrit, " [a]u sein de la sphère marchande, nous avons constaté le 'paradoxe de Carnegie', selon lequel, pour réussir en affaires, il faut non seulement valoriser l'autre, mais le faire 'sincèrement' ${ }^{20}$. Mais Godbout semble limiter ce paradoxe à l'activité économique. Or, on peut penser qu'il en va de même pour l'amour et l'amitié. Pour profiter de l'amour, il faut le vivre avec sincérité ; vivre avec sincérité ici élimine l'acteur égoïste, calculateur et motivé par ses intérêts. Par conséquent, toutes les

\footnotetext{
${ }^{18}$ Michel Bauer, op. cit., p. 140.

19 Michel Amiot, op. cit., p. 216.

${ }^{20}$ Jacques T. Godbout, L'esprit du don, Paris, La Découverte, 1992, p. 140.
} 
personnes qui expriment leur motivation pour une vocation, une cause, qui dépeignent leur amour du métier, ne peuvent être considérées simplement comme utilitaristes ou comme étant guidées par un simple raisonnement. En fait, la critique utilitariste, malgré ses réserves, exprime la nécessité de tenir compte de la situation multidimensionnelle de l'acteur et de prendre en considération l'émotion aussi bien que la raison, chaque fois qu'il faut comprendre une action humaine, même une action économique.

La critique du paradigme de l'intérêt s'avère essentielle à une compréhension plus adéquate de l'action humaine. Certaines et certains analystes de la théorie des choix rationnels ont semblé privilégier l'élargissement de la définition de la rationalité pour combler les lacunes inhérentes aux difficultés engendrées par l'utilisation de la notion étroite de rationalité. Aujourd'hui, les sciences sociales s'accordent pour dire que cette "forme faible ", c'est-à-dire la conception économique néoclassique de la rationalité, laisse échapper trop d'informations. Selon Caillé, l'utilisation de la "forme forte de la rationalité » permet de théoriser l'action humaine. Ainsi, la théorie des choix rationnels n'est possible que par l'acceptation de cette forme forte de la rationalité qui comporte, elle aussi, comme on l'a montré, d'énormes difficultés au niveau d'une vérification empirique. Comme Caillé, encore, l'indique,

[d]ans sa version forte, en effet, l'hypothèse de rationalité ne procède d'aucun fondement empirique déterminé [...] [1]a science économique se déploie tout entière à partir de la prise au sérieux de cette version forte dont elle entend tirer toutes les conséquences et dégager toutes les implications. ${ }^{21}$

En somme, que ce soit la forme forte de la rationalité ou que ce soit une rationalité limitée, il n'en demeure pas moins que les modèles s'appuient sur le principe de la rationalité de l'acteur pour expliquer son action, sur une notion de rationalité qui, d'une part, par cette extension conceptuelle, ne peut plus vraiment être définie strictement comme rationalité et qui, d'autre part, n'assume pas l'intrication de l'émotion et de la rationalité. L'action est le produit de relations multiples et le résultat d'un dispositif complexe qui font qu'elle est moins un choix qu'une suite conséquente.

${ }^{21}$ Alain Caillé, La démission des clercs, op. cit., p. 112. 


\section{Méthodologie}

Le but fondamental de cette recherche est de faire état du réductionnisme du modèle utilitariste dans l'explication de l'action humaine. Ce paradigme centre l'ensemble de ses théories sur une rationalité de l'acteur, une rationalité suffisamment significative pour fournir une explication à l'action humaine. Cet objectif, cependant, n'est pas le seul. Il y en a trois autres : 1) mettre en lumière la complexité de l'action ; 2) montrer l'inhérence de l'émotion et de la raison dans l'action; 3) inscrire l'action dans une perspective relationnelle.

\subsection{Hypothèses fondamentales à vérifier}

Cette recherche a pour but de mettre en lumière les limites de la théorie qui tient l'acteur pour rationnel. Dans sa praxis, l'acteur est essentiellement émorationalité. La psyché se révèle dans la dialectique qu'il entretient avec les structures sociales. En fait, la rationalité de l'acteur n'est qu'un des éléments de la dynamique dont procède l'action. Cette recherche entend démontrer que l'axiomatique de l'intérêt abuse de la notion de rationalité de l'acteur, un acteur conçu comme rationnel, intentionné et libre. L'utilitarisme affirme l'existence :

- d'un acteur rationnel en tant qu'il envisage, dans toutes ses actions, chacune des possibilités qui s'offrent à lui dans le but de satisfaire ses intérêts. Or, si l'acteur répond à ses intérêts, on devrait être en mesure d'observer des gains associés à chacune de ses actions et une stratégie pour qu'il parvienne à ses fins ;

- d'un acteur intentionné en ce sens qu'il décide de ses actions pour qu'elles correspondent à des stratégies toujours antérieures à l'action. Or, si l'acteur agit avec intention, chacune d'elles a été prévue ;

- d'un acteur libre et autonome puisqu'il décide librement de ses actions. Or, si l'acteur est libre et autonome, il peut agir différemment à tout moment.

Partant de ces constats, toute recherche qui veut faire état de l'utilisation métonymique de ces éléments dans l'explication habituelle de l'action doit faire état d'actions typiques quotidiennes vécues par des individus et interprétées par eux. En découvrant le sens que ces acteurs donnent à leurs actions et, selon leurs explications, on verra bien si ces postulats ont encore quelque pertinence. 
Les données recueillies sont donc destinées à confronter ces postulats utilitaristes à l'empirie. Elles doivent aussi servir à mettre en évidence la dimension émotionnelle de chacune des actions. L'action est, en principe, et rationnelle, et émotionnelle. Si l'être humain est émorationnel, l'analyse devrait montrer que l'action contient toujours une dimension rationnelle et une dimension émotionnelle : rationnelle lorsque l'acteur agit dans le cadre d'informations plus ou moins analytiques ; émotionnelle lorsque chacune des relations indique une émotion et où l'information n'intervient pas de façon strictement analytique. De plus, ces données doivent faire voir que l'action ne peut être comprise que dans le relationnel. Le modèle explicatif de l'action doit concevoir l'action dans la relationalité.

\subsection{Collecte des données}

L'analyse s'appuie sur trente entrevues (numérotées de 4 à 33). Chacune d'elles porte sur des actions passées ou présentes qui ont une incidence dans la vie de tous les jours. Pour arriver à mieux saisir les éléments constitutifs de l'action, les répondants s'expriment sur la manière par laquelle ils en sont venus à s'inscrire dans les diverses activités. La démarche permet d'accumuler des données sur les facteurs qui ont mené à l'action. Elle se veut ouverte à la complexité de ces vécus, dans une optique que nous pourrions qualifier de " postrationalisante » en ce qu'elle n'entend pas se contenter d'une modélisation utilitariste où l'acteur a, en principe, déjà rationalisé l'entièreté de son action. Le terme « utilitaire» renvoie à la part de rationalité de l'action ou à la vision d'une action conçue essentiellement comme rationnelle. En jouant sur les mots, nous pouvons dire que nous demandons à l'acteur de postrationaliser, c'est-à-dire d'examiner les éléments conscients et inconscients qui l'ont mené à s'inscrire dans une activité. Ce n'est pas dire qu'une explication postrationnelle de l'acteur fournit l'ensemble des facteurs qui sont intervenus dans l'action. En fait, nombreux sont ceux qui ne semblent pas questionner l'origine de leurs actions ou les facteurs qui y ont contribué.

\subsection{Entrevues}

Les entrevues sont de type semi-dirigé. Cette technique donne beaucoup de champ à l'intervieweur en même temps qu'elle centre les informations ${ }^{22}$; en outre, elle permet de respecter la spécificité de chacun des répondants qui, ainsi, livre des informations qui correspondent littéralement à sa perception.

${ }^{22}$ Sylvain Giroux, Méthodologie des sciences bumaines : la recherche en action, Saint-I Laurent (Québec), Éditions du Renouveau pédagogique, 1998. 
Le protocole d'entrevue sollicite deux genres d'information. Une première section comporte des questions de type sociodémographique qui sont posées sous une forme directive. Dans une seconde section, la personne interviewée divulgue les activités dans lesquelles elle est ou était impliquée. Compte tenu du protocole, l'entrevue se déroule en deux temps. Dans un premier temps, les participants doivent répondre aux questions d'ordre sociodémographique, puis élaborer la liste des diverses activités dans lesquelles ils sont ou ont été impliqués. Dans un deuxième temps, l'intervieweur reprend chacune des activités identifiées par l'interviewé et lui demande de s'exprimer librement sur la manière par laquelle il en était arrivé à les mettre en œuvre.

Les entretiens ${ }^{23}$ ont permis d'obtenir de l'information sur les diverses activités de chacun des acteurs, autant au niveau de l'emploi qu'en ce qui a trait aux activités familiales, bénévoles et sociales. Les personnes interrogées ont pu s'exprimer pour décrire comment elles en sont arrivées à s'impliquer dans chacune de ces activités.

\section{4. Échantillon}

La collecte de données a été effectuée selon une technique non probabiliste. Il s'agit d'une sélection formée de répondants que nous avons choisis de façon plutôt accidentelle en tentant d'obtenir de l'information d'acteurs provenant de tous les secteurs de la communauté.

Par ailleurs, nous croyons que des variables comme la culture, l'ethnie, les croyances religieuses, l'instruction, le sexe, la langue, l'âge... n'ont pas de pertinence pour l'analyse qui est ici entreprise et que l'action de tout être humain est le produit d'un dispositif complexe et unique à un moment précis de son histoire. Toutefois nous avons utilisé certaines de ces variables pour contrôler l'indifférence de ces variables. C'est pourquoi, il est prétendu que, pour vérifier l'hypothèse, le choix des acteurs comme échantillon n'a pas d'importance. Pas plus d'ailleurs qu'il n'en aurait pour l'utilitariste qui prétend que son explication est universelle, donc, qu'elle ne varie selon aucun facteur.

Les entretiens ont eu lieu dans une communauté du nord de l'Ontario. L'échantillon se compose de onze femmes et de dix-neuf hommes dont la moyenne d'âge est de quarante-cinq ans. Tous sont francophones. Vingt-trois des trente répondants sont mariés et vingt-quatre ont

${ }^{23}$ Les données proviennent de trente entrevues d'une durée qui varie de 45 à 90 minutes. Des rendez-vous ont été fixés à l'avance et l'intervieweur s'est rendu à l'endroit désigné par l'interviewé. Tous les entretiens, sauf un, n'avaient lieu qu'avec une seule personne; dans un des entretiens, le conjoint de la personne interrogée s'est joint à la discussion. 
des enfants. Les occupations sont variées : huit se définissent comme entrepreneurs, cinq sont en éducation, quatre sont journaliers, quatre sont en communication, quatre encore sont des personnes au foyer, deux se disent gérant d'entreprise, une personne travaille dans la fonction publique, une autre fait de la pastorale, une dernière est à la retraite.

\section{Analyse de contenu}

Les verbatim des trente entrevues constituent le corpus de l'analyse. Dans un premier temps, la recherche vise, en tentant de découvrir les facteurs qui ont mené l'acteur à participer à différentes activités, à présenter une analyse selon les postulats de la rationalité économique. L'instrument de collecte de données doit être en mesure de permettre de dégager ce que le chercheur, dans une perspective économiciste, présenterait comme résultat. Par la suite, l'analyse dégage les facteurs qui sont négligés par cette approche et, dans une analyse du second degré, fait valoir les actions qui ne relèvent pas d'un simple agencement logique.

Cette analyse comporte deux niveaux de catégorisation qui permettent, d'une part, d'en arriver à la construction d'un système catégoriel et, d'autre part, à fournir des résultats essentiels à la grille d'interprétation nécessaire compte tenu des buts de cette recherche.

\subsection{Catégorisation}

La catégorisation s'effectue à deux niveaux. Dans les deux niveaux, elle se réalise « avec une grille d'analyse ouverte où il n'existe pas de catégories [classes] analytiques au départ ; les catégories [classes] sont alors induites des textes analysés $»^{24}$.

Dans le premier niveau, une grille de catégorisation permet de placer les énoncés selon le domaine d'activités et selon certaines classes déterminées par induction. Dans cette première tentative, il s'agit de prendre les quatre grands domaines d'activités (famille, emploi, bénévolat et passe-temps) que l'on trouve dans le questionnaire d'entrevue et d'inscrire tous les énoncés des verbatim dans les classes

${ }^{24}$ Réjean Landry, "L'analyse de contenu », in Benoît Gauthier (dir.), Recherche sociale. De laproblématique à la collecte des données, Presses de l'Université du Québec, $3^{\circ}$ édition, 1997, p. 329-356. 
suivantes : contexte, événements, prédisposition historique, structures (socialisation, obligations morales et familiales), circonstances, métaphysique (spriritualité, croyances religieuses, cartomancie...), relation et réseau, irrationalité, rationalité, défi (vocation, cause, amour du métier) et autres. De plus, à chacune de ces grilles s'ajoutent des tableaux indiquant les gains et les pertes liés aux diverses activités. Cette catégorisation s'est avérée peu efficace puisqu'on peut regrouper plusieurs classes pour, finalement, les réduire à six. Un autre problème lié à cette catégorisation est que l'on applique ces classes à des domaines d'activités plutôt qu'à des activités spécifiques. Par exemple, dans le domaine du bénévolat, le répondant peut s'adonner à plus d'une activité mais son explication peut être différente d'une activité à l'autre.

Dans cette première analyse, on a pu observer que les répondants centralisent leurs explications sur une des dimensions de l'ensemble de la situation.

En général, les acteurs centrent leur discours sur les activités qui sont les plus significatives pour eux à un moment de leur vie. Par exemple, une dame investit, présentement, tout son temps dans son entreprise. Elle parle seulement de cette activité. Pourtant, elle a élevé trois enfants qui sont sûrement encore très importants dans sa vie (bien que maintenant partis de la maison), elle avait un emploi à temps plein, elle a déménagé à plusieurs reprises, etc. Tout le vécu de cette femme fait que, présentement, ce travail est ce qu'il y a de plus significatif dans sa vie.

On remarque que certains individus soit se limitent à raconter les faits, soit apportent une explication plus approfondie, en ajoutant les émotions et autres facteurs qui définissent le cadre de l'action.

L'analyse montre aussi que, dans une postrationalisation, et cela même chez ces répondants qui fournissent une explication plus éclairée de leurs situations, on n'est qu'en partie conscient des éléments qui mènent à une action. En fin de compte, cette observation ne fait que confirmer l'hypothèse de cette recherche. L'acteur expose, par son explication, les limites de sa rationalité dans la rationalisation de son action. L'analyse révèle la complexité de ce qui compose l'action humaine.

Une deuxième tentative de catégorisation consiste à théoriquement distinguer les diverses actions auxquelles on attribue chaque fois six classes: vécu, structures, relations, défis, événements et métaphysique. Nous disons 


\section{NPSS, VOLUME 1, NUMÉRO 2, 2006}

bien théoriquement puisqu'il est impossible, dans la pratique, de vivre ces activités de façon exclusive. Après l'analyse de quelques entrevues, il fut de plus en plus difficile de caser une classe dans une action. Par exemple, dans un énoncé où le répondant dit qu'il aime aider les gens, il est approprié de placer cette information dans les classes : vécu, contexte et relations. Il devint évident que les structures, le contexte, le vécu et les relations font partie intégrante de toute action. De plus, il fallut constater qu'il est impossible de dissocier chacune des classes. L'action est dans un même temps structure, vécu, contexte, relation, émotion, vocation, défi. La frontière entre les classes est floue et les classes ne sont pas empiriquement exclusives ; elles n'ont été données que pour des fins méthodologiques. À chaque instant, les actions à titre de parent, de conjoint, de collègue, d'employé, que dépeignent les répondants peuvent difficilement être dissociées les unes des autres. Tous ces mondes ${ }^{25}$ se produisent synchroniquement, l'acteur est tous ses mondes en même temps, dans des structures et dans un contexte précis. Par exemple, pour un répondant, la conjointe devient gravement malade. L'ensemble des actions de cette personne est affecté : que ce soit l'emploi, les activités sociales et, évidemment et surtout, les activités familiales. Cet acteur doit nécessairement agir, maintenant, dans l'intérêt de l'autre ; ce n'était certainement pas une intention et il est difficile de parler de liberté et d'autonomie. Dans la situation d'un père de famille de trois enfants, il est impossible d'exclure d'une analyse les limites qui sont associées à cette réalité ; l'analyse de ses activités hors famille demande qu'on en comprenne la complexité.

Deux visées sont atteintes avec cette catégorisation. Premièrement, nous avons pu nous imprégner profondément de chacune des entrevues pour ainsi être en mesure d'en dégager par abstraction les éléments communs à l'ensemble des répondants. Ces abstractions révèlent des éléments à l'intérieur desquels s'explique l'action. Nous retenons cinq classes qui réapparaissent à chacune des entrevues : 1) structure familiale, 2) vécu et valeurs, 3) circonstances, 4) causes sociales et 5) sollicitation d'autrui et conseils de personnes influentes. Deuxièmement, cet exercice nous a permis de passer à une autre forme de catégorisation de l'information recueillie. Le tableau 1 présente les mondes dans lesquels les répondants agissent.

${ }^{25}$ Luc Boltanski et I aurent Thévenot, De la justification. Les économies de la grandeur, Paris, Gallimard, essais, 1991. 


\section{Tableau 1 \\ Mondes dans lesquels s'inscrivent les répondants}

Différents mondes

\section{Famille}

Emploi

Église

Personnes âgées

Municipalité

Organisations sociales

Causes sociales

Clubs sportifs

Comités dans la communauté

Jeunes

Autres
Description de l'activité dans laquelle les répondants sont inscrits ou étaient inscrits

inscrit dans une famille (grands-parents, parent, enfant, sœur, frère)

occupe ou a occupé un emploi rémunéré

est attaché à l'église ou organise des activités liées à la religion

s'occupe de personnes âgées, à la maison ou dans les foyers pour personnes âgées

donne de son temps à la politique municipale

participe aux activités des Filles d'Isabelle, des Chevaliers de Colomb, du club Richelieu...

défend des causes qui lui tiennent à cœur. Par exemple, la pénurie de médecins, les soins pour personnes âgées, les droits des francophones, la survie de la communauté...

participe comme membre, observateur, joueur, entraîneur...

fait partie du comité de la salle paroissiale, du comité de la St-Jean, du comité de développement économique...

organise des activités pour les jeunes

s'implique dans la Société du cancer, dans les activités pour les personnes qui ont des défis mentaux et physiques, en contribuant, par exemple, à des campagnes de levée de fonds...

Au second niveau de catégorisation, nous disposons certaines données dans deux tableaux fondamentaux : un premier, révélant la complexité des mondes de chacun des répondantes et des répondants (tableau 2) et, un second, exposant les situations qui soulignent la difficulté à recourir à la notion d'autonomie (tableau 3). Les tableaux 2 et 3 révèlent le degré d'implication du répondant dans chacune de ses activités.

Le tableau 2 permet de comptabiliser les activités dans lesquelles l'acteur évolue. Le système de catégorisation utilise onze classes pour inscrire chacune des activités des répondants. 
86 NPSS, VOLUME 1, NUMÉRO 2, 2006

Tableau 2

Complexité des divers mondes

\begin{tabular}{|c|c|c|c|c|c|c|c|c|c|c|c|}
\hline \multirow[b]{2}{*}{ 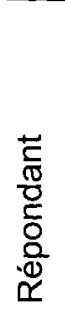 } & \multirow[b]{2}{*}{$\begin{array}{c}\stackrel{\Phi}{\overline{\bar{E}}} \\
\stackrel{\mathbb{\varpi}}{\Perp}\end{array}$} & \multirow[b]{2}{*}{$\frac{\bar{o}}{\frac{\varrho}{E}}$} & \multicolumn{9}{|c|}{ Bénévolat } \\
\hline & & & 忍 & 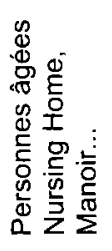 & 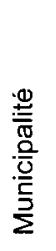 & 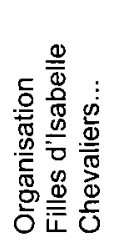 & 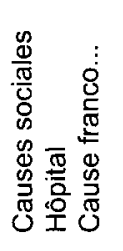 & 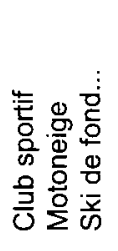 & 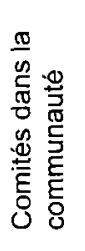 & $\begin{array}{l}\stackrel{\$}{\Phi} \\
\stackrel{5}{\leftrightarrows}\end{array}$ & 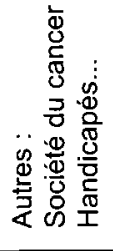 \\
\hline 04 & I & & $\mathrm{GI}$ & 1 & & $\mathrm{Gl}$ & 1 & $\mathrm{Gl}$ & & & \\
\hline 05 & 1 & 1 & & $\mathrm{GI}$ & & 1 & & & Gl & & GI \\
\hline 06 & GI & $\mathrm{Gl}$ & TGI & & 1 & & $\mathrm{GI}$ & & TGI & $\mathrm{GI}$ & I \\
\hline 07 & TGI & 1 & $\mathrm{Gl}$ & TGI & & 1 & I & I & 1 & & 1 \\
\hline 08 & 1 & $\mathrm{TGI}$ & & TGI & & & & & & & \\
\hline 09 & $\mathrm{Gl}$ & I & & $\mathrm{Gl}$ & & & & & & & I \\
\hline 10 & GI & TGI & & $\mathrm{GI}$ & 1 & & $\mathrm{Gl}$ & & I & TGI & \\
\hline 11 & 1 & 1 & & & $\mathrm{GI}$ & $\mathrm{Gl}$ & & $\mathrm{Gl}$ & 1 & 1 & \\
\hline 12 & $\mathrm{Gl}$ & 1 & & & & & 1 & & 1 & & \\
\hline 13 & $\mathrm{Gl}$ & $\mathrm{Gl}$ & & & 1 & & 1 & 1 & & $\mathrm{Gl}$ & \\
\hline 14 & 1 & 1 & 1 & & & & & & I & 1 & I \\
\hline 15 & I & I & & & & & & & & & \\
\hline 16 & 1 & 1 & & & 1 & & GI & 1 & Gl & & 1 \\
\hline 17 & 1 & $\mathrm{TGI}$ & $\mathrm{GI}$ & & & 1 & TGl & & $\mathrm{Gl}$ & TGI & GI \\
\hline 18 & $\mathrm{Gl}$ & 1 & & & & 1 & 1 & 1 & & & 1 \\
\hline 19 & $\mathrm{Gl}$ & I & 1 & 1 & 1 & 1 & GI & 1 & & & \\
\hline 20 & $\mathrm{TGI}$ & 1 & $\mathrm{Gl}$ & I & 1 & 1 & 1 & TGI & & GI & \\
\hline 21 & 1 & $\mathrm{Gl}$ & TGI & 1 & I & Gl & & & 1 & TGI & GI \\
\hline 22 & TGI & $\mathrm{Gl}$ & $\mathrm{Gl}$ & I & 1 & $\mathrm{Gl}$ & 1 & I & $\mathrm{TGI}$ & & 1 \\
\hline 23 & $\mathrm{Gl}$ & $\mathrm{Gl}$ & I & 1 & & 1 & TGI & 1 & I & & 1 \\
\hline 24 & 1 & $\mathrm{Gl}$ & & & & 1 & & 1 & & & \\
\hline 25 & 1 & 1 & & & & & & 1 & & & \\
\hline 26 & $\mathrm{Gl}$ & GI & $\mathrm{Gl}$ & I & & I & & 1 & GI & & \\
\hline 27 & $\mathrm{Gl}$ & GI & $\mathrm{Gl}$ & & & & GI & GI & 1 & 1 & 1 \\
\hline 28 & TGI & TGI & $\mathrm{Gl}$ & & & I & & & 1 & & 1 \\
\hline 29 & 1 & 1 & & & 1 & & $\mathrm{GI}$ & & I & & \\
\hline 30 & 1 & $\mathrm{TGI}$ & & & & & $\mathrm{TGI}$ & 1 & 1 & & \\
\hline 31 & TGI & $\mathrm{Gl}$ & $\mathrm{GI}$ & 1 & & 1 & 1 & 1 & 1 & $\mathrm{Gl}$ & 1 \\
\hline 32 & 1 & 1 & & 1 & & & & $\mathrm{Gl}$ & I & & \\
\hline 33 & $\mathrm{GI}$ & TGI & I & 1 & $\mathrm{Gl}$ & & & & & & \\
\hline
\end{tabular}

«I ॥ Implication dans l'activité ; « GI » Grande implication ; « TGI » Très grande implication 


\section{PIERRE BOUCHARD / THÉORIE DEL'ACTION ET PARCOURS DEVIE}

Un deuxième tableau essentiel à cette recherche indique les situations où il appert que la notion d'autonomie est peu adéquate pour qualifier la réalité (tableau 3). Le système de catégorisation permet d'identifier les situations qui limitent la liberté des personnes dans leurs actions. Celles-ci sont : famille (parent, conjoint et enfants vivent en famille), maladie (le répondant souffre d'une maladie qui limite ses possibilités d'action), maladie d'un proche (le répondant doit s'en occuper, ce qui exige du temps et de l'énergie), emploi (le répondant occupe un emploi), bénévolat (le répondant donne de son temps à des organismes), activités sociales (le répondant consacre du temps à des activités sociales comme le sport, les sorties et les passe-temps).

Le tableau 3 fait aussi ressortir le degré d'implication qu'exigent ces situations. Il permet d'observer les situations qui restreignent les possibilités d'action au moment de l'entrevue, mais aussi tout au long de la vie. Il s'agit de vérifier la transformation dans les situations au cours de la vie des répondants.

Avec ce tableau, on constate que les situations « famille » et " maladie d'une personne proche " se transforment de façon significative, mais que, pour les autres situations, il y a peu de changements. Au niveau de « la famille ", au cours de la vie, les individus passent de jeune adulte, à parent, pour ensuite, vivre le départ des enfants du foyer. À chaque étape, la famille subit de grands changements. Pour ce qui est de la « maladie d'une personne proche ", le décès de cette personne est souvent ce qui occasionne le changement.

Les tableaux 2 et 3 suffisent à mettre en évidence la complexité associée au caractère multiple de la situation dans laquelle vit l'acteur et ils restreignent en eux-mêmes la portée des concepts de rationalité, d'intention et de liberté dans l'explication de l'action humaine à un point où l'explication n'a plus d'appui théorique.

\subsection{Catégories retenues}

Cette section se penche plus directement sur les propos des personnes interrogées. Lorsque nous avons demandé aux sujets comment ils expliquent leur implication dans diverses activités, ils ont décrit explicitement et implicitement comment cela était arrivé. L'analyse des entrevues permet de comprendre a posterioni les facteurs qui conduisent à l'activité. On est en mesure, en effet, de percevoir des éléments constitutifs de l'action le contexte, les circonstances, les relations, le vécu -, le tout pénétré d'une émorationalité. 
88 NPSS, VOLUME 1, NUMERO 2, 2006

Tableau 3

Situations soulignant la difficulté de l'usage de la notion d'autonomie

\begin{tabular}{|c|c|c|c|c|c|c|c|c|c|c|c|c|}
\hline \multirow{2}{*}{$\begin{array}{c}\text { Répondants } \\
4\end{array}$} & \multicolumn{2}{|c|}{$\begin{array}{c}\text { Famille } \\
\text { conjoint et enfant }\end{array}$} & \multicolumn{2}{|c|}{$\begin{array}{l}\text { Maladie } \\
\text { répondant }\end{array}$} & \multicolumn{2}{|c|}{$\begin{array}{c}\text { Maladie } \\
\text { personne proche }\end{array}$} & \multicolumn{2}{|c|}{ Emploi } & \multicolumn{2}{|c|}{ Bénévolat } & \multicolumn{2}{|c|}{ Activités sociales } \\
\hline & Ciii & $C \mathrm{P}$ & I & I & I & & & & TGI & TGI & I & I \\
\hline 5 & $\mathrm{C}_{i i}$ & C P & I & $\mathbf{I}$ & I & & GI & & TGI & TGI & I & I \\
\hline 6 & $\mathrm{C}_{i i}$ & C? & I & I & & & TGI & TGI & TGI & TGI & I & I \\
\hline 7 & $\mathrm{C}_{\text {iiniiiii }}$ & $C D$ & I & I & TGI & & GI & & TGI & I & GI & I \\
\hline 8 & $\mathrm{C}_{\mathrm{iji}}$ & $C P$ & & & & & I & TGI & & & & \\
\hline 9 & $\mathrm{C}_{\mathrm{ii}}$ & $\mathrm{C}_{i i} \mathrm{P}$ & & & GI & & I & 1 & & & & \\
\hline 10 & $\mathrm{C}_{\mathrm{ii}}$ & $\mathrm{C}_{i i} \mathrm{P}$ & I & $\mathrm{I}$ & GI & & TGI & TGI & TGl & TGI & TGI & I \\
\hline 11 & $\mathrm{C}_{\mathrm{iii}}$ & $\mathrm{C}_{i i i} \mathrm{P}$ & & & & & I & I & GI & GI & GI & GI \\
\hline 12 & $\mathrm{C}_{\mathrm{i}}$ & $\mathrm{C}_{\mathrm{j}} \mathrm{P}$ & & & & & I & I & GI & I & I & \\
\hline 13 & $\mathrm{C}_{\mathrm{i}}$ & $C_{i} D$ & Gl & & GI & & GI & GI & TGI & 1 & TGI & I \\
\hline 14 & $\mathrm{C}_{i i}$ & $\mathrm{C}_{\mathrm{i}} \mathrm{P}$ & I & 1 & I & I & 1 & I & I & I & 1 & I \\
\hline 15 & $\mathrm{C}$ & C P & & & I & I & I & 1 & & & 1 & I \\
\hline 16 & & $P$ & & & I & I & GI & $\mathrm{GI}$ & GI & GI & GI & GI \\
\hline 17 & $\mathrm{C}_{\text {iiiii }}$ & $?$ & & & & & TGI & TGI & TGI & TGI & GI & I \\
\hline 18 & & $\mathrm{P}$ & & & & & I & 1 & I & I & TGI & TGI \\
\hline 19 & $\mathrm{C}_{i i i i}$ & C $\mathbf{P}$ & & & I & I & Gl & $\mathrm{GI}$ & GI & GI & GI & GI \\
\hline 20 & $\mathrm{C}_{i j i}$ & $\mathrm{C}_{i i i} ?$ & & & & & I & I & TGI & $\mathrm{TGI}$ & TGI & TGI \\
\hline 21 & & $?$ & & & & & I & GI & TGI & TGI & GI & GI \\
\hline 22 & $C_{i i}$ & C P & I & I & GI & GI & $\mathrm{Gl}$ & GI & TGI & TGI & GI & GI \\
\hline 23 & $\mathrm{C}_{\mathrm{iiii}}$ & $\mathrm{CP}$ & & & GI & GI & GI & GI & TGI & TGI & I & I \\
\hline 24 & $\mathrm{C}_{\mathrm{iiii}}$ & $\mathrm{C}_{i i i i} \mathrm{P}$ & & & & & GI & $\mathrm{Gl}$ & I & I & GI & GI \\
\hline 25 & & $P$ & & & & & I & I & & & TGI & TGI \\
\hline 26 & $C_{i i i}$ & C? & & & & & GI & GI & GI & GI & GI & GI \\
\hline 27 & $C_{i i}$ & $\mathrm{C}_{i i} \mathrm{P}$ & & & I & 1 & GI & GI & GI & GI & $\mathrm{Gl}$ & I \\
\hline 28 & $\mathrm{C}_{i i i}$ & $C_{i i i} P$ & & & & & GI & GI & GI & GI & I & I \\
\hline 29 & $\mathrm{C}_{i i}$ & $?$ & & & & & I & I & I & I & GI & $\mathrm{Gl}$ \\
\hline 30 & $\mathrm{C}$ & C P & & & & & TGI & TGI & GI & GI & I & 1 \\
\hline 31 & $C_{i i j i}$ & $C_{j} P$ & I & I & I & & TGI & TGI & GI & GI & I & GI \\
\hline 32 & $C_{i i i}$ & $\mathrm{C}_{i j} \mathrm{P}$ & & & I & & I & I & GI & 1 & TGI & GI \\
\hline 33 & $\mathrm{C}_{\text {iiiii }}$ & C P & & & & & TGI & TGI & TGI & TGI & I & 1 \\
\hline
\end{tabular}

\section{Légende}

Sous chacune des catégories, la première colonne indique l'implication au cours de la vie ; la seconde montre l'implication actuelle de l'acteur. «P $»$ parents qui occupent encore une place importante dans la vie des répondants ; «C » présence d'un conjoint ; « $i$ » enfant qui demeure encore à la maison ; «?» information non disponible ; « $\mathrm{D}$ » décès du conjoint ou des parents ; « $\mathrm{I}$ » Implication ; «GI » Grande implication ; «TGI » Très grande implication. 


\section{PIERRE BOUCHARD / THÉORIE DELACTION ET PARCOURS DEVIE}

\subsection{Grille d'interprétation}

Pour vérifier l'hypothèse selon laquelle l'utilitarisme abusc du conccpt dc rationalité dans l'explication de l'action humaine, il apparait pertinent de soumettre à quelque vérification les principes suivants : l'acteur répond à ses intérêts, l'acteur agit par intention, l'acteur est un être libre et autonome. Si l'acteur agit pour satisfaire ses intérêts, par intention et qu'il est libre et autonome, il est, par conséquent, un être rationnel. Ainsi le veut la vision utilitariste. Pour examiner la pertinence de ces principes, nous avons recouru à une grille d'interprétation de laquelle se dégagent huit composantes qui sont, en réalité, des tâches analytiques et que nous pouvons résumer de la façon suivante :

L'acteur répond à ses intérêts :

- identifier dans l'activité si l'acteur répond à ses intérêts ;

- dégager les cas où se révèle l'irrationalité de l'action ;

- repérer l'émotion dans l'action.

L'acteur agit par intention:

- révéler les situations où l'acteur ne peut pas avoir d'intention;

- indiquer les circonstances qui mènent à l'action ;

- présenter les situations où l'on a sollicité la participation de l'acteur ou encore où l'acteur réagit relativement aux besoins d'un groupe ou d'une situation.

L'acteur est libre et autonome :

- montrer les activités où l'acteur agit pour satisfaire les intérêts des autres ;

- repérer les diverses activités de l'acteur qui supposent le respect de règles et d'obligations (mettre en lumière la complexité des différents mondes de l'acteur).

Mais avant de modéliser ainsi les données, il faut jeter son regard sur les données recueillies.

\subsubsection{Description des données}

\subsubsection{Intrication des raisons invoquées par les acteurs}

Interrogé à savoir pourquoi il faisait un travail difficile où les heures étaient longues, ne recevant qu'une faible compensation financière pour ce travail, le jeune répondant 25 déclare : 
c'est une question que je me suis demandée ; c'est pour aider ; c'est probablement pour aider; je ne vois pas d'autre raison. So I guess que l'argent, ça me disait pas rien [...]. J'adorais ça ; je ne sais pas pourquoi, là ; mais j'adorais ça ; on travaillait, on travaillait, on commençait à 6 heures le matin, puis on finissait à 10 heures le soir, puis c'était comme une journée, une journée de 8 heures.

Comme dans cet exemple, dans la majorité des cas, l'activité trouve à sa source, en premier lieu, du plaisir, de la satisfaction, du défi, de l'amour du métier. On observe que l'explication des interviewés, pour l'ensemble des actions, est liée à l'émotion. Au départ, les sujet eux-mêmes indiquent qu'ils agissent par émotion. Plusieurs lient leurs actions à leur vécu, à leurs valeurs et à leurs croyances. Cependant, peu d'entre eux élaborent explicitement sur le contexte, la période historique, la structure. Pourtant, chaque situation montre l'intrication de ces éléments, qui, dans leur dynamique, se transforment perpétuellement pour produire un dispositif unique à chaque instant.

Le répondant 6 a accepté une réduction de salaire, une perte de vacance et d'avantages sociaux pour se lancer dans un travail inconnu sans réellement en connaitre la teneur. Le répondant 33 prend une retraite pour se lancer dans une entreprise touristique qui exige énormément de temps et d'énergie, et ce, en entraînant avec lui sa conjointe. Dans une perspective utilitariste, ce couple aurait sûrement profité de prendre une retraite complète plutôt que de s'aventurer dans une telle activité communautaire. Un professeur s'exprime sur toutes ses années en enseignement et explique que chaque jour de sa carrière a été un défi. Pour lui, le défi a constitué le principal élément de l'explication. Les résultats montrent que la profession d'enseignant fait appel à cette dimension émotionnelle qui permet de poursuivre la carrière. De plus, ce même homme dirige et coordonne le fonctionnement d'un organisme communautaire depuis plus de trente ans où il s'acharne à offrir un service essentiel à la communauté alors qu'il exprime que « la reconnaissance, bien... c'est pratiquement nul ». Dans une compilation utilitaire des gains et des pertes, les pertes dépassent largement les gains. Il en est de même pour chaque personne interrogée, dans l'ensemble de ses activités. Un répondant travaille comme journalier, où il n'apprécie guère le travail à l'usine, mais soulève qu'il aime la stabilité, la rémunération, les quarts de travail qui lui donnent beaucoup de temps libre pour ses autres activités ainsi que les vacances que lui procure cet emploi. On constate que la sécurité et la stabilité de l'emploi sont une dimension de l'acteur qui ne peut pas être séparée des autres qui sont liées à d'autres activités. Cet homme s'implique au niveau de la municipalité où il donne énormément de son temps. Le répondant 19 révèle: «Puis j’ai vraiment aimé la construction, comme j’adore 
ça, même si c'est excessivement exigeant, puis difficile [...] ; puis on a souvent des problèmes ; comme la construction en général, c'est pas le domaine qu'on fait de l'argent quand tu es... tu es petit ». Encore une fois, le calcul utilitaire des gains et des pertes montre un large déficit. Il en est de même pour l'ensemble des activités bénévoles. Les gens donnent de leur temps et de leur argent pour contribuer à l'offre de services dans leur communauté.

\subsubsection{Grandes thématiques}

Si l'on tente de regrouper dans quelques grandes métacatégories la plupart des éléments qui se révèlent dans le discours des acteurs, on obtient les thématiques suivantes: structure familiale, vécu et valeurs, s'émouvoir pour des causes sociales, circonstances et sollicitation d'autrui.

\subsection{Structure familiale}

La famille joue un rôle primordial dans l'action pour l'ensemble des répondants. On observe que, lorsque la structure familiale se transforme, les membres de la famille vivent aussi de grands changements, ce qui réduit énormément le champ de possibilités d'action. La répondante 31 est restée à la maison pendant quinze ans. Elle s'est occupée de quatre enfants. Les enfants ont grandi et elle a plus de temps de disponible et songe à aller sur le marché du travail. Une occasion d'acheter un commerce lui a permis de se lancer en affaires tout en prenant soin de ses enfants. De plus, l'entreprise a fourni une occasion aux enfants d'acquérir de l'expérience de travail. Pendant de nombreuses années, les enfants ont travaillé dans l'entreprise. Après que les enfants aient quitté le foyer pour faire des études, elle et son conjoint envisagent de vendre le commerce. La répondante songe à passer à autre chose. Toutes ces actions sont liées à la structure familiale. De même, le répondant 33 a aussi ouvert une entreprise pour créer de l'emploi pour ses enfants. Une fois que les enfants ont quitté la maison, il vend le commerce. Le répondant 17 a travaillé comme enseignant pendant trente-cinq ans. Tout au long de sa carrière, il donne énormément de son temps aux activités parascolaires. À travers tout cela, son épouse et lui élèvent des enfants. À sa retraite, le couple se sépare. Il exprime que, maintenant qu'il est seul, il peut plus que jamais s'impliquer dans divers projets communautaires. Cependant, il mentionne aussi qu'il s'assure que les jeunes qui travaillent avec lui et qui commencent une carrière professionnelle prennent du temps avec leur jeune famille. La répondante 18 est maintenant entrepreneure parce que ses parents à la retraite ont légué aux enfants les entreprises familiales. La répondante 27 a enseigné dans une institution postsecondaire durant quelques années. L'institution ferme ses portes, mais la répondante pourrait 
continuer à servir l'école, si elle déménage. Son conjoint a un bon emploi et elle demeure dans la région. Elle tente un retour aux études, mais son enfant malade exige trop de temps et d'énergie. Il est évident, ici, que la structure familiale oriente l'action. Chacune des entrevues permet de tirer des exemples qui ont trait à ce phénomène.

La structure familiale correspond aussi à des possibilités d'actions qui sont créées par l'environnement de l'acteur, c'est-à-dire que, dans de nombreux cas, l'acteur peut agir de telle ou telle manière parce que son conjoint ou un autre membre de sa famille lui permet de disposer de temps pour s'adonner à telle activité. Par exemple, si le répondant 11 est entré en politique, c'est parce que sa conjointe restait à la maison avec les trois enfants. Un autre a acheté un commerce parce que la mère pouvait gérer le commerce tout en prenant soin de leurs enfants. Une répondante gère un logement pour personnes âgées pendant que son mari travaille à l'extérieur; ses enfants sont tous partis de la maison. Le répondant 19, qui est obligé de subvenir financièrement aux besoins de sa famille, ajoute des heures à sa journée, ce qui n'est possible que parce que sa conjointe reste à la maison à prendre soin des enfants.

Dans le même ordre d'idées, la relation de couple a aussi une grande importance. Plusieurs personnes ont indiqué qu'elles s'adonnaient à telle activité parce qu'elle leur permettait de faire quelque chose en couple. Une répondante se fait offrir un poste à l'extérieur de la ville. Elle refuse parce que son conjoint a un emploi stable qu'il aime et laisse ainsi tomber l'occasion d'évoluer dans une carrière qu'elle aime beaucoup.

Une plus jeune répondante abandonne tous ses projets d'études et de carrière pour revenir se marier. Cette jeune femme avait pour projet, quelques mois auparavant, de poursuivre des études postsecondaires et d'entreprendre une carrière professionnelle.

Il est évident que la structure familiale a un effet important sur l'action. Elle limite les possibilités et, indubitablement, ne conduit pas à des actions dont les justifications pourraient être qualifiées d'utilitaires.

\subsection{Vécu et valeurs}

Les acteurs vivent tous les jours des expériences de vie qui les transforment. Ce bagage, ces expériences forment leur vécu. Les données indiquent que ce vécu a une grande influence sur l'action. Plusieurs en soulignent l'influence. Par exemple, les répondants 17 et 22 évoquent l'époque de leurs études au Collège des Frères. Pour d'autres, c'est la vie familiale qui est riche de signification. Une répondante qui, très jeune, avait apprécié le tissage, s'est jointe, des années plus tard, à un groupe de tisserandes. 
On observe que l'action est souvent le produit de valeurs et de principes qui suscitent une émotion. Pour l'ensemble, les valeurs familiales sont très importantes ; pour plusieurs, le travail est un principe fondamental ; pour d'autres, c'est la musique ; pour d'autres encore, c'est l'éducation. Ces valeurs et ces principes orientent les actions. L'acteur agira en respectant ses valeurs et ses principes. Mais ces valeurs et ces principes sont le produit du vécu de l'acteur. Pour le répondant 22, « il faut absolument que tu les vives, tes principes, puis les valeurs. C'est quelque chose que tu cultives constamment; puis, les valeurs, bien, c'est les valeurs chrétiennes de la famille, étant l'une des plus importantes ». Pour le répondant 33, "[l]e travail, c'est à peu près ce qu'il y a de plus important dans la vie ». Ce même répondant parle du sens du devoir. Les données indiquent clairement que le vécu et les valeurs acquises orientent l'action.

\subsection{S'émouvoir pour des causes sociales}

Dans chacune des entrevues, les individus indiquent qu'ils s'adonnent à une activité parce qu'ils y ont été incités par une cause qui leur tient à coeur, par émotion.

\section{Tableau 4}

Actions provoquées par une cause sociale

\begin{tabular}{|c|c|c|}
\hline Domaines & Répondant(e)s & Réaction aux différents systèmes \\
\hline Éducation & $\begin{array}{l}5,6,9,10,13,15,16,17 \\
19,21,22,27,30,33\end{array}$ & $\begin{array}{l}\text { - établir des services pour les enfants qui } \\
\text { éprouvent des difficultés d'apprentissage } \\
\text { établir un système pour accommoder les } \\
\text { décrocheurs scolaires } \\
\text { - } \quad \text { ouvrir un centre d'éducation alternatif pour } \\
\text { adultes } \\
\text { développer un programme de musique } \\
\text { (chorale, fanfare, etc.) } \\
\text { développer un nouveau cursus pour tout le } \\
\text { système primaire et secondaire } \\
\text { assurer l'apprentissage des élèves }\end{array}$ \\
\hline Santé & $\begin{array}{l}4,5,6,7,8,9,1210,13 \\
18,21,23,27,31,33\end{array}$ & $\begin{array}{l}\text { - } \quad \text { améliorer le système pour les personnes âgées } \\
\text { - } \quad \text { fournir un appui aux comités pour les } \\
\text { différentes maladies (cœur, cancer, reins...) }\end{array}$ \\
\hline Justice & $\begin{array}{l}5,6,7,10,12,14,17,21 \\
22,23,27,29,30,31\end{array}$ & $\begin{array}{l}\text { - défendre les droits des victimes des agressions } \\
\text { - } \quad \text { asexuelles } \\
\text { - } \quad \text { lutter pour les droits des Franco-Ontariens }\end{array}$ \\
\hline Gouvernement & $\begin{array}{l}5,6,8,10,11,14,16,17 \\
20,22,25,33\end{array}$ & $\begin{array}{l}\text { critiquer la bureaucratie et le système de } \\
\text { financement gouvernemental }\end{array}$ \\
\hline Culturel & $\begin{array}{l}6,7,10,11,12,13,14,16 \\
17,18,19,20,21,23,24 \\
26,27,29,30,31,32,33\end{array}$ & $\begin{array}{l}\text { - } \quad \text { lutter pour les services en français } \\
\text { organiser des activités sociales et sportives } \\
\text { pour le développement des enfants }\end{array}$ \\
\hline
\end{tabular}




\section{NPSS, VOLUME 1, NUMÉRO 2, 2006}

Les répondants 6, 9, 10, 17 et 22 travaillent à fournir de l'aide aux gens qui ne semblent pas s'intégrer au système d'éducation actuel. Le répondant 22 s'exprime ainsi : « [o]n aurait aimé être plus libres dans notre enseignement, puis être plus innovateurs, mais le système ne nous le permettait pas, nous bloquait constamment ». De même, le répondant 17 dit que « [v]ous avez dans vos écoles des programmes qui sont extraordinaires, puis vous arrivez à un résultat médiocre. Avec des programmes extraordinaires vous réussissez la médiocrité ". Les répondants 12 et 30 mentionnent que le système d'éducation dirige les élèves sans pour autant tenir compte des besoins de l'individu. Le répondant 19 indique que le système d'éducation dénigre les métiers comme ceux qui sont liés à la construction, n'enseigne pas le respect du travail ouvrier.

D'autres critiquent le système judiciaire. La répondante 14 est frustrée par les droits qu'on donne aux agresseurs sexuels. Certains déplorent le fait que le système semble produire des citoyens de plus en plus individualistes. Les répondants 5, 6, 10,11,13,17,22, 31 et 33 dénoncent le manque d'implication des gens dans leur communauté. Pourtant, chacun est impliqué ou s'est impliqué dans sa communauté à une période de sa vie.

Les répondants $16,17,23$ et 33 questionnent les politiques et le fonctionnement des gouvernements. Pour les répondants $4,5,7,8$ et 33 , le système pour les personnes âgées ne répond qu'en partie aux besoins de cette population. D'autres encore, les répondants 6 et 10, portent un jugement sévère sur le système de santé qui, dans une communauté majoritairement francophone, ne dessert pas encore la population dans sa langue : ces individus travaillent au recrutement de nouveaux médecins pour la communauté.

On constate que les gens s'impliquent sincèrement dans la cause qu'ils ont à cœur. Ils croient fermement dans ce qu'ils font ; que ce soit le chef pompier, le propriétaire d'une maison pour personnes âgées, le directeur de la chorale, ceux qui ont collaboré à la politique municipale, les enseignants, les professionnels en communication, tous démontrent un sincère dévouement. Comme le mentionne le répondant 22, qui est enseignant, "[] es conditions de travail d'enseignant sont enviables, mais si tu n'aimes pas les enfants, tu ne peux pas penser en faire un emploi ».

\subsection{Circonstances}

Les circonstances constituent aussi un élément important pour rendre compte de l'action. Si l'acteur a posé cette action, c'est dû à des événements souvent anodins qui ont eu lieu au cours de la vie. Des circonstances aussi 
insignifiantes qu'une rencontre au centre commercial, une émission à la télévision, un appel d'un ami peuvent transformer le cours d'une vie. Lorsque les événements ouvrent une possibilité d'action qui correspond à son vécu, on parle d'occasion, et c'est de là que vient l'apparence d'intention. Il est certain que le modèle normatif que produit la théorie des choix rationnels arrive à des résultats qui, parfois, semblent correspondre à l'intention de l'acteur, sauf qu'il aurait pu en être autrement et, à ce moment, on aurait fourni une autre explication de l'action. De plus, l'action peut sembler le résultat d'intentions, mais les étapes pour y accéder ont exigé de modifier les actions tout au long du parcours. Enfin, si l'individu agit sur les structures sociales qui le déterminent, cet acteur influence l'orientation de ses actions. Plus encore, la personne vit des événements majeurs qui dirigent son action.

Dans l'analyse des données, on observe que l'ensemble des acteurs vivent des événements marquants au cours de leur vie. Si l'on s'arrête à quatre événements majeurs de la vie d'un acteur, on constate que, dans l'échantillon, $23 \%$ des individus ont eu une maladie qui les a obligés à changer leurs habitudes ; $50 \%$ ont un parent proche qui est malade et qui demande une attention et des soins de leur part ; $30 \%$ ont vécu le deuil d'au moins une personne proche; et, enfin, plus de $87 \%$ ont subi des changements imprévus au niveau de l'emploi.

Il est intéressant d'examiner le parcours de certains répondants, plus précisément les changements imprévus au niveau de l'emploi ou de la carrière. Parmi ces modifications d'orientation, nous relevons les suivantes : d'enseignant à directeur en communication; de président du syndicat à contremaître; d'études en biologie à maîtrise en histoire; de boucher à journalier à l'usine de pâte à papier; de bûcheron à mécanicien, à ouvrier, à entrepreneur; de femme au foyer à entrepreneure; de mécanicien à bûcheron; de vendeuse dans un garage à vendeuse dans un magasin de meubles; de journalier dans une usine à responsable de l'entretien d'un terrain de golf; de propriétaire de restaurant à propriétaire d'une papeterie. Les reconversions les plus surprenantes ne peuvent certainement pas être les conséquences d'intention.

Quels qu'ils soient, les changements provoqués par ces événements ont transformé le cours de la vie, non pas seulement de l'individu même, mais aussi de tout son environnement. $\mathrm{Si}$, maintenant, on s'intéresse à d'autres événements importants comme un changement de carrière, un déménagement, une nouvelle relation, on peut affirmer que chacune des personnes interrogées subit à chaque jour, et tout au long de sa vie, des événements qui la mènent à agir comme elle l'a fait sans pour autant en avoir jamais eu l'intention. 
Comment expliquer le cas de cet homme qui se rend à l'usine pour se chercher un emploi comme journalier et qui se trouve, à la fin de la journée, comme opérateur d'équipements lourds ? On devrait être en mesure d'expliquer aussi que, pendant de longues années, ce même individu représente les membres de son syndicat, pour ensuite accepter un poste de contremaitre pour la même compagnie. Dans ces deux fonctions, cet homme agit avec sincérité et dévouement. Comment expliquer le cas d'un enseignant du secondaire qui quitte un emploi stable et bien rémunéré pour relever le défi d'un poste comme agent de communication dans un institut financier? Comment expliquer le cas d'une femme peu instruite, ne parlant pas l'anglais, qui réussit à gérer un commerce sur la place publique et qui en vient à s'intéresser aux soins palliatifs? Les entrevues montrent que l'action des gens est souvent le résultat de concours de circonstances. Les acteurs, suite à des circonstances bien précises, se trouvent dans des activités qu'ils n'auraient jamais pu prévoir.

Dans cette même thématique, on peut aussi inclure le contexte ou la période historique qui expliquent en partie l'action. Quelques-uns ont mentionné que, lorsqu'ils ont quitté l'école, il était facile d'obtenir un emploi, qu'on pouvait facilement changer d'emploi. Par ce fait, l'acteur était moins incité à poursuivre des études. Il y a aussi les valeurs familiales, plus traditionnelles, que certains ont conservées et qui jouent un grand rôle. Il y a encore une communauté en transformation, comme c'est le cas dans la région étudiée où les francophones se sont affichés dans tous les domaines: les médias, la politique, l'éducation, l'économie, le social, la culture, la santé, etc.

\subsection{Sollicitation d'autrui et conseils de personnes influentes}

Une autre observation importante : le nombre d'actions qui sont le produit de la sollicitation d'une autre personne. Chacun des répondants a expliqué son implication dans des activités par le fait qu'une autre personne avait sollicité sa participation. On trouve ce phénomène autant en relation à l'emploi qu'en ce qui se rapporte au bénévolat ou aux loisirs. À la question «Comment en êtes-vous arrivé à cette activité ? », tous les répondants donnent comme explication qu'on leur en a fait la demande. On indique que "ils m’ont demandé », " une amie m’a demandé », " un tel m’a demandé ».

On observe aussi que, dans de nombreux cas, les interviewés ont agi selon les conseils de personnes influentes. Par exemple, des répondants ont dit avoir été incités à agir de telle manière. Quelques-uns ont indiqué que les 
curés étaient responsables de leurs orientations, soit qu'ils les encourageaient, soit qu'ils les renvoyaient du Collège des Frères. D'aucuns ont pris une autre direction suite au commentaire d'une collègue : un stagiaire en enseignement s'est fait dire par sa superviseure qu'il n'était pas fait pour l'enseignement. Il a donc laissé l'enseignement pour un an. Pourtant cet homme a fait dix-sept ans d'enseignement dans une école secondaire. D'autres ont suivi la direction que suggéraient les orienteurs scolaires.

\subsubsection{Résumé}

Après observation, on constate que les thématiques «structure familiale », " vécu et valeurs » et « circonstances » se trouvent dans toute action alors que "s'émouvoir pour une cause sociale » et "sollicitation d'autrui » apparaissent dans chacune des entrevues, mais pas dans chacune des actions. Cependant, on note que la thématique «s'émouvoir pour une cause sociale » s'inscrit dans l'élément « vécu et valeurs » alors que «sollicitation d'autrui » s'intègre à l'élément " circonstances ». La structure familiale, le vécu et les valeurs, puis les circonstances forment donc une structure inhérente à chacune des actions (voir schéma 2).

\section{Schéma 2}

Structure des éléments explicatifs de l'action

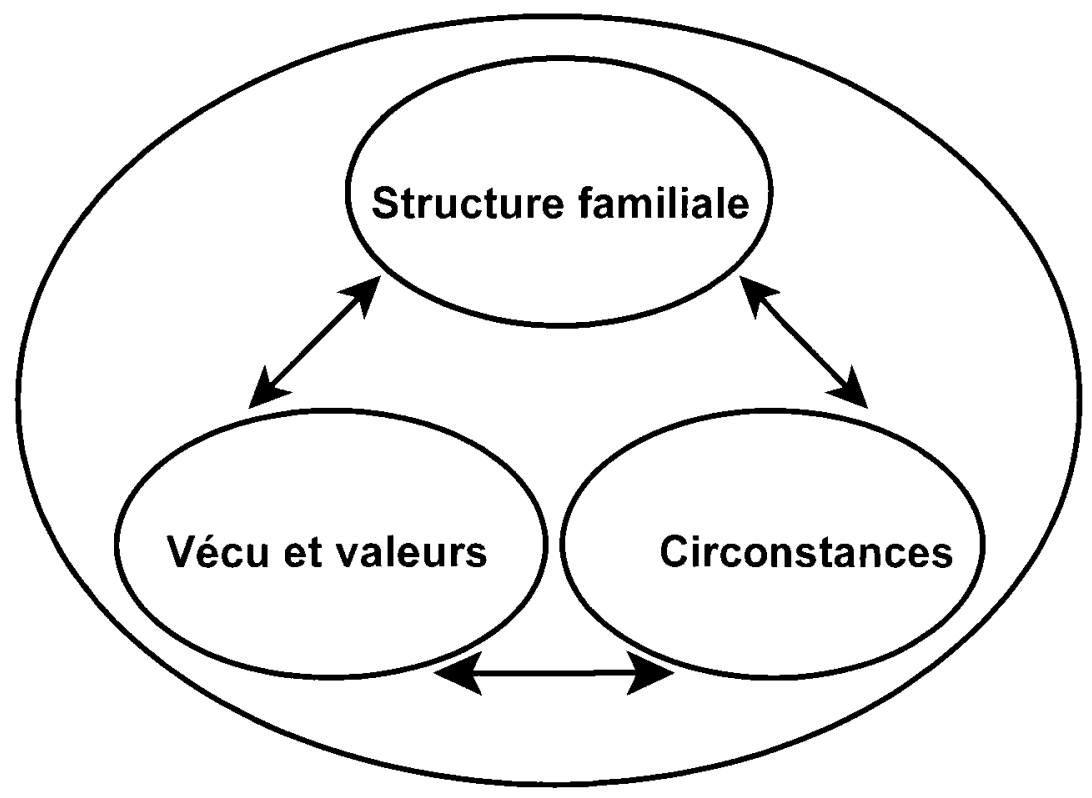


Cette structure illustre la complexité de l'action. L'action est toujours une combinaison de ces éléments.

Ces premières observations mettent en lumière certains éléments constitutifs de l'explication de l'action du point de vue des acteurs. Leur intrication témoigne d'une réalité complexe. Maintenant, il s'agit de rassembler ces traits pour répondre aux questions que la problématique a laissées en suspens.

\subsubsection{Interprétation des données}

Selon la théorie des choix rationnels, entre autres, l'acteur agit nécessairement pour satisfaire à ses intérêts, c'est-à-dire qu'il vise toujours à maximiser ses gains ou, pour le moins, à minimiser ses pertes. Cette quête utilitaire suppose un acteur rationnel. Toutefois, les données d'entrevues font voir que, souvent, l'acteur, dans une activité concrète, semble comptabiliser plus de pertes que de gains. De plus, dans bien des cas, les activités répondent beaucoup plus aux intérêts des autres qu'à ceux de l'acteur lui-même.

Les informations qu'on peut tirer des deux niveaux de catégorisation servent à vérifier cette sous-hypothèse en permettant :

- d'identifier dans l'activité si l'acteur répond à ses intérêts ;

- de dégager les cas où se révèle l'irrationalité de l'action ;

- de repérer l'émotion dans l'action.

\subsubsection{Acteur et intérêts}

$\mathrm{Si}$, selon l'utilitarisme, l'acteur cherche toujours à maximiser ses gains, nos observations devraient montrer que l'individu a pris en considération toutes ses possibilités avant de poser une action et qu'il est en mesure d'en évaluer les conséquences en pertes et en gains. Une rationalité utilitaire pure supposerait que l'être humain est régi essentiellement par de l'intérêt et on devrait observer que ses décisions sont prises en considération de tous les choix possibles, du moins de ceux dont il peut avoir conscience.

Comment arriver à déterminer que l'acteur tient compte de toutes ses possibilités? Cela peut se faire en présentant les gains et les pertes liés à cette activité. Cela peut aussi se faire en observant comment il en est arrivé à cette activité selon qu'il indique qu'elle est une série circonstancielle d'événements ou qu'elle est due à l'influence de quelqu'un : orienteur, parent ou autre. 
Prétendre que l'acteur agit en fonction de ses intérêts suppose qu'il a estimé chacune de ses possibilités pour retenir celle qui lui procurera un maximum de gains au moindre coût. Il parait important d'apporter certaines précisions sur le concept d'intérêt. L'utilitariste semble faire une distinction entre trois formes d'intérêt : l'intérêt financier, l'intérêt personnel (glorification, avancement personnel...) et l'intérêt lié à la satisfaction (le plaisir, le bonheur, le défi, l'amour du métier...). Tout d'abord, on peut s'interroger à savoir si la satisfaction doit être considérée comme intérêt. Ensuite, on doit se demander comment ne pas dire que l'intérêt personnel n'est pas satisfaction? Et, enfin, on peut se questionner sur la manière de prétendre que l'intérêt financier n'est pas aussi satisfaction? Le point commun de ces trois formes d'intérêt est la satisfaction, ce qui, en fait, est une émotion.

Dans leurs propos, les interviewés mentionnent que leur motivation première est souvent cet intérêt, dit satisfaction. Quand on leur demande pourquoi ils se sont inscrits dans telles activités, ils répondent : par plaisir ( J'aime ça », «C'est le fun», "C'est plaisant »...), par défi (entreprendre un nouvel emploi, établir de nouveaux services, développer de nouvelles techniques, apporter du soutien à un organisme...) et par amour du métier ( II n'y a pas plus beau métier », " c'est la plus belle des professions »...).

Les théories d'inspiration économiciste soutiennent que l'acteur comptabilise les gains et les pertes pour obtenir un résultat dont l'action est le moyen, c'est-à-dire qu'une analyse des gains et des pertes devrait permettre d'expliquer l'action. Cependant, ce calcul exige qu'on accorde une valeur comptable à des intérêts personnels et à des intérêts de satisfaction. Pour comptabiliser, pour comparer, il est nécessaire d'accorder des valeurs à des intérêts pour ainsi produire des modèles théoriques de l'action. Or, ces intérêts appartiennent strictement à l'acteur et sont, par ce fait, uniques à cet individu. Ces valeurs proviennent de cet acteur socialisé et sont le construit de ses relations et de ses déterminants. Malgré ce débat, on cherche à identifier l'intérêt comme origine explicative de l'action.

\subsubsection{Gains et pertes}

Dans la plupart des activités, l'intérêt lié à la satisfaction semblait le gain principal. Le plaisir, le défi et l'amour du métier constituent des facteurs importants dans l'explication de l'action. Incidemment, peu de répondants ont soulevé l'aspect financier comme intérêt dans leurs activités, et ce, même au niveau de l'emploi. Des répondants ont quitté des emplois stables et bien rémunérés pour se lancer dans des carrières ou des emplois qui souvent demandaient d'accepter une baisse de salaire et un surcroit de 
temps et d'énergie. De plus, il est encore plus difficile de concevoir les implications familiales et bénévoles autrement que par l'émotion. Comment alors comprendre que le dévouement et la participation à une activité s'ajoutent à une journée au travail ? Comment le chercheur peut-il prétendre connaitre la dimension utilitaire de la satisfaction de cet acteur dans une telle action?

De plus, l'analyse des entrevues fait état de différences au niveau du temps et de l'énergie qui sont liées aux diverses activités. Pour certains, c'est avec la famille ; pour d'autres, c'est au travail ; pour d'autres, encore, c'est dans les activités sociales. Pour l'utilitariste, l'acteur ne fait que satisfaire à ses intérêts. Comment le chercheur peut-il expliquer ces distinctions s'il fait strictement un calcul froid des gains et des pertes? En fait, l'acteur vit de l'émotion dans l'ensemble des situations qui le concernent, qu'il s'agisse d'économie, de famille ou de vie sociale. On a plutôt affaire à un dispositif complexe qui est lié à la situation existante. Malgré ces dénonciations, l'utilitarisme suppose un acteur rationnel qui pose un regard analytique sur l'ensemble de sa réalité, même de son vécu.

Par ailleurs, plusieurs personnes interviewées expriment leur nonconscience au moment où elles ont entrepris diverses activités. Dans une perspective rationnelle, l'acteur devrait, en principe, avoir examiné les plus et les moins d'une activité. Toutefois, on observe que les personnes se sont impliquées dans quelque chose et que ce n'est que par après qu'elles en ont découvert les conséquences. Elles se sont dites naïves lorsqu'elles se sont engagées. Les énoncés suivants témoignent de la part d'irrationalité de l'acteur dans des actions.

Tableau 5

Exemples d'énoncés exprimant la non-conscience des choix possibles

Énoncés

Répondant

" c'était toutes des choses que j'ai réalisées, une fois que j'étais rendu ici »

" y avait un tas de choses comme ça, [...] dont je n'étais pas conscient quand j'ai pris ma décision de partir»

« quand je suis devenu conscient de la mission [...], j'ai vu quelque chose qu'on pouvait atteindre, puis, même, j'en étais complètement ignorant avant d'embarquer »

" Je suis partie en treizième année pour aller à l'université, je n'avais aucune idée pourquoi je m'en allais à l'université "

« Next thing you know, je déménage... "

«Et puis tu ne décides pas ça de toi- même [...] c'est un cheminement presque inconscient, là ; et tu t'embarques là dedans, puis tu suis la vague comme on dit...» 
Il est difficile de parler d'une action rationnelle lorsque plusieurs décisions importantes ont été prises après avoir consulté une cartomancienne, ou en s'en remettant à la providence, à des croyances religieuses et à des convictions. Ces cas indiquent que l'acteur ne possédait pas l'ensemble de l'information et qu'il ne pouvait pas faire un choix rationnel. Ils indiquent aussi que les actions sont bien plus souvent le produit de l'action elle-même que la conséquence d'une réflexion antérieure.

Tous les individus qui ont agi en sentant le besoin d'offrir des services qui faisaient défaut à la communauté se sont lancés dans des projets dans lesquels il était impossible d'entrevoir l'avenir. Ces exemples montrent que les acteurs entreprennent des activités sans en connaitre réellement les implications. Dans ces cas, il est difficile de prétendre que l'acteur avait évalué les gains et les pertes.

Contrairement à ce que prétendent les utilitaristes, nos données montrent que l'acteur s'implique dans des actions, pour l'essentiel, de façon inconsciente, ce qui affaiblit la théorie des choix rationnels et réduit l'apport de la rationalité comme postulat dans l'explication de l'action. Autrement dit, même si la personne possédait certaines informations au moment de l'action, elle ne pouvait comprendre la situation que de façon très approximative. Il ne s'agit pas simplement ici de rationalité limitée au sens de Simon, mais surtout de Boudon qui entend qu'on maximise les gains et minimise les pertes dans un contexte circonstanciel d'informations; la rationalité est davantage limitée en ce qu'elle ne joue qu'un faible rôle dans la psyché à partir de laquelle, ou dans laquelle, ou avec laquelle l'activité est entamée. Si l'acteur avoue qu'il n'était pas conscient des exigences de l'activité, il est difficile de concevoir l'action simplement sous l'éclairage d'une rationalité utilitaire. La réflexion $a$ posteriori met bien en évidence le caractère absurde de la thèse qui veut que tout ait lieu de façon intentionnelle suite à une décision purement rationnelle.

\subsubsection{Complexité des mondes}

L'individu vit à tous les instants des situations multiples, ce qui oblige à se questionner sur son niveau de rationalité en situation. Si l'acteur vit dans une multitude de mondes en même temps, il ne peut appréhender sa réalité que de façon diffuse. Selon le paradigme utilitariste, pour optimiser ses intérêts, le sujet doit être en mesure de prendre en considération chacune des possibilités qui sont associées à cette situation. Si l'être humain vit des situations multiples, il ne peut pas agir en fonction de son intérêt dans chacune de ses situations. En fait, le caractère multisituationnel de la réalité d'un acteur social montre qu'il agit en ne tenant compte que de certains éléments, tous à la fois rationnels et émotionnels. 
Les données montrent que l'acteur est impliqué dans différentes activités (tableau 2). Chacune de ces activités impose des règles et des obligations. Il est évident que des intérêts dans une activité peuvent entrer en contradiction avec les intérêts dans une autre activité. On constate que c'est souvent le cas entre les obligations familiales et l'emploi. Cependant, on observe que la possibilité de s'adonner à diverses activités a pour cause l'ensemble des relations de l'acteur, de ses divers champs d'intervention. Si l'individu peut s'absenter de la maison, c'est qu'il peut compter sur d'autres relations, par exemple celle qu'il entretient avec un conjoint, pour subvenir aux besoins familiaux.

Il semble important de distinguer comment les acteurs se sont impliqués dans une activité et ce qui fait qu'ils continuent à s'y adonner. Les données démontrent que l'implication de l'acteur dans une activité s'explique surtout par des causes extérieures : le contexte, la sollicitation d'autrui et les circonstances sont les facteurs les plus couramment évoqués. Pourquoi maintenant persiste-t-il dans l'activité ? Il appert que le plaisir, le défi et l'amour du métier, mais aussi les obligations et les attentes constituent des facteurs importants pour chacune des actions. Ces facteurs sont liés à l'émotion. Par conséquent, il est essentiel de reconnaître l'inhérence de l'émotion et de la rationalité dans l'action ; et que cette émorationalité est le résultat de relations, qu'elle est un vécu complexe qui s'est construit et qui se construit à travers l'ensemble des relations, tout au long de la vie.

\subsubsection{Acteur et intention}

La théorie rationalisante prétend que l'acteur agit de façon intentionnelle. L'acteur agit de telle manière parce qu'il en a l'intention. Si l'acteur s'adonne à une activité, c'est qu'il en a eu l'intention. La grille d'analyse, pour vérifier cette hypothèse, prescrit les tâches suivantes : révéler les situations où l'acteur ne pouvait pas avoir d'intention et présenter les situations où l'on a sollicité la participation de l'acteur ou encore où l'acteur réagissait relativement aux besoins d'un groupe ou d'une situation.

\subsection{Action vue comme circonstancielle}

L'action est souvent le produit de circonstances. Les circonstances obligent l'acteur à réagir. Ces circonstances font partie du quotidien et de la construction de la satisfaction de l'acteur. Tous les répondants ont connu au cours de leur vie des événements qui exigeaient d'eux qu'ils s'ajustent à la situation, à la nouvelle réalité. De plus, plus d'un événement circonstanciel rendent difficile leur interprétation par évocation de la thèse de l'intention. 
L'étude permet d'observer que, dans l'ensemble, l'action n'est pas produite intentionnellement, qu'elle est plutôt l'effet de circonstances. Des événements comme la maladie, un décès, un emploi, la rencontre d'une personne constituent des éléments plus ou moins importants qui peuvent transformer l'ensemble d'une vie. Dans chacune des entrevues, les circonstances rendent compte de l'action. Par exemple : l'acteur vit des événements imprévus (maladie, décès d'un proche, échec à une élection...), change complètement de carrière (vingt-six individus ont fait état de ce type de transformation), agit par rapport aux circonstances qui ont ouvert des occasions, répond à des attentes.

\subsection{Sollicitation ou conseil d'autrui}

Dans l'ensemble, les individus interviewés mentionnent que leurs actions sont suscitées par une demande. Lorsqu'on les interroge sur la manière dont ils se sont engagés, la majorité répondent en disant : «ils m'ont demandé », « ils sont venus nous chercher », " [une telle personne] m'a demandé », etc. On observe que l'acteur agit fréquemment suite à la sollicitation d'une autre personne.

Dans les entretiens, plusieurs personnes indiquent qu'elles ont suivi les recommandations d'orienteurs, de collègues, d'amis. Ces gens influents, censés comprendre l'acteur mieux qu'il ne se comprend lui-même, ont guidé l'action d'une manière qui, souvent, ne correspond même pas à l'acteur. C'est, par exemple, le cas de personnes qui ont suivi les recommandations des orienteurs de l'école secondaire ; c'est aussi le cas d'individus qui ont entrepris des études et qui, au bout d'un certain temps, se sont aperçus que cette voie ne leur convenait pas du tout. On constate que leur trajectoire a été dictée en extériorité. Ces cas n'indiquent pas que l'action est le résultat d'un choix. On observe que des personnes influentes jouent un rôle important dans l'explication de l'action.

La notion d'intention a quelque chose d'intéressant puisque, dans certains cas, les actions peuvent sembler en être les résultantes. Intention renvoie tautologiquement à préférences; alors les acteurs sont rationnels pour autant qu'ils aient l'intention de ce dont ils ont l'intention. Il s'agit plutôt de comprendre et de répéter que « les acteurs socialisés agissent sur les structures qui les déterminent $»^{26}$. Il est évident que si l'acteur agit dans une certaine direction, il est possible qu'il se trouve dans une activité qui pourrait sembler intentionnelle. 


\section{4

\subsubsection{Acteur libre et autonome}

Que suppose être un acteur libre et autonome? À l'extrême, un acteur libre pourrait changer d'emploi, changer de relation, changer de vie quand bon lui semble. Il n'en est pas ainsi dans la réalité et le tableau 6 l'illustre très clairement. Au départ, l'acteur vit dans une société qui lui impose des règles, explicitement et implicitement. On parle du code criminel, du code routier, du code familial... et de tous les systèmes tacites de règles. De plus, dans sa vie privée, l'acteur se voit aussi imposer des obligations qui limitent sa liberté d'agir. Chacune des activités auxquelles participe la personne est soumise à des règles morales et elle est restreinte par sa nécessaire inscription dans la socialité.

La grille d'analyse pour examiner cette question comporte les directives suivantes : repérer les activités de l'acteur qui exigent le respect de règles et d'obligations ; montrer les activités où l'acteur agit pour satisfaire aux intérêts des autres ; indiquer les circonstances qui mènent à l'action.

\subsection{Liberté et situations de vie}

Le tableau 6 illustre les situations dans lesquelles les répondants sont impliqués et qui restreignent sa liberté d'action. On y trouve les classes suivantes : famille, maladie du répondant, maladie d'une personne proche (conjoint, enfant, parent), emploi, bénévolat et activités sociales. On observe que tous les acteurs ont des obligations dans les divers mondes de leur quotidien qui réduisent, pour l'ensemble, leur liberté d'action et que, de plus - ce que les données de cette recherche ne permettent pas d'établir clairement -, l'ensemble des répondants ont, vraisemblablement, au cours de leur vie, eu des engagements financiers qui ont aussi limité leurs actions.

Tableau 6

Situations qui limitent la liberté des individus

\begin{tabular}{cccccc}
\hline $\begin{array}{c}\text { Famille } \\
\text { conjointe / } \\
\text { conjoint et enfants }\end{array}$ & $\begin{array}{c}\text { Maladie de la } \\
\text { répondante / } \\
\text { répondant }\end{array}$ & $\begin{array}{c}\text { Maladie d'une } \\
\text { personne proche }\end{array}$ & Emploi Bénévolat & Activités sociales \\
\hline 30 & 8 & 15 & 27 & 26 & 27 \\
$100 \%$ & $27 \%$ & $50 \%$ & $90 \%$ & $87 \%$ & $90 \%$ \\
\hline
\end{tabular}

Les données révèlent d'importantes transformations au niveau de la famille sans, pour autant, réduire l'apport de cette dimension de l'action pour chacun des répondants. La famille constitue le lieu d'activités le plus 


\section{PIERRE BOUCHARD / THÉORIE DE L'ACTION ET PARCOURS DE VIE}

commun dans l'échantillon. On note aussi que $27 \%$ des répondants vivent ou ont vécu une maladie grave qui a exigé une transformation du mode de vie. On observe que $50 \%$ des individus ont dû réorganiser leur vie en rapport avec la maladie d'un proche. Ces situations restreignent la liberté d'action. Enfin, on remarque que la presque totalité des personnes gèrent différents mondes en même temps, ceux de la famille, de l'emploi, du bénévolat et des activités sociales, c'est-à-dire qu'elles jouent, en principe, avec des règles explicites et implicites dans ces quatre mondes. L'acteur, en fait, évolue simultanément dans ces quatre mondes.

Le tableau 3 montrait aussi que les acteurs, souvent, présentent un même enthousiasme à participer aux diverses activités de leur vie. On remarque que les acteurs qui se sont impliqués dans le bénévolat, alors qu'ils étaient jeunes, vont toujours faire du bénévolat. De même, lacteur qui s'implique passionnément dans son travail le fera pour chacun des emplois qu'il occupera. Les activités changent, mais la passion pour les activités ne semble pas se modifier.

Du même coup, ces données mettent en lumière la complexité de l'action (tableaux 2 et 3). Toutes ces obligations ne sont pas exclusives. L'acteur a des engagements moraux et sociaux dans chacune de ses activités, en plus d'avoir des responsabilités envers sa famille, son emploi et les organismes ou les causes auxquels il se consacre. Dans l'action, on suppose une rationalité, mais cette action est le résultat complexe d'éléments qui constituent un dispositif de l'action. À cette action se greffent des limites et des restrictions qui rendent irréel le principe d'une simple rationalité.

\subsection{Activités qui répondent aux intérêts des autres}

L'acteur agit souvent davantage en fonction des intérêts des autres que des siens. La grille utilisée permet d'identifier onze domaines différents d'activités.

En examinant ces mondes (voir tableaux 2 et 3), on remarque que, dans bien des situations, l'acteur répond aux intérêts des autres beaucoup plus qu'aux siens. On constate que chacun des répondants satisfait aux intérêts des autres ${ }^{27}$ dans au moins trois activités, et certains des répondants se trouvent dans plus de neuf activités sur les onze identifiées. Les répondants sont impliqués en moyenne dans sept domaines de cet ordre.

${ }^{27}$ Intérêt des autres est ici utilisé dans la logique utilitariste ; nous parlerions plutôt de relations. 


\section{Tableau 7 Activités en fonction des intérêts des autres}

\begin{tabular}{|c|c|}
\hline Famille & $\begin{array}{l}\text { l'acteur répond aux intérêts du conjoint, des enfants, de } \\
\text { parents }\end{array}$ \\
\hline Emploi & l'acteur dépasse les demandes de l'employeur \\
\hline Église & l'acteur organise des activités à l'église \\
\hline Personnes âgées & l'acteur donne de son temps pour les personnes âgées \\
\hline Municipalité & l'acteur donne de son temps au conseil municipal \\
\hline Organisme & $\begin{array}{l}\text { l'acteur est impliqué dans un ou plusieurs organismes qui } \\
\text { ont comme objectif d'apporter de l'aide aux plus démunis }\end{array}$ \\
\hline Cause sociale & $\begin{array}{l}\text { l'acteur est impliqué dans la défense d'une cause (hôpital, } \\
\text { école, francophonie) }\end{array}$ \\
\hline Club sportif & $\begin{array}{l}\text { l'acteur s'adonne comme joueur à des activités sportives ou } \\
\text { collabore à l'organisation des activités dans les différents } \\
\text { clubs sportifs }\end{array}$ \\
\hline Comité dans la communauté & $\begin{array}{l}\text { l'acteur s'est joint à divers comités (Centre pour jeunes, Centre } \\
\text { pour déficients mentaux, Chambre de commerce, comité de la } \\
\text { salle paroissiale, etc.) }\end{array}$ \\
\hline Organisme pour les jeunes & $\begin{array}{l}\text { l'acteur organise des activités pour les jeunes (entraîneur, } \\
\text { carnaval d'hiver, surveillant, etc.) }\end{array}$ \\
\hline Autres & $\begin{array}{l}\text { l'acteur donne de son temps à divers organismes (Société du } \\
\text { cancer, handicapés, droit des victimes d'agressions sexuelles, } \\
\text { etc.) }\end{array}$ \\
\hline
\end{tabular}

Le tableau 8 révèle que la famille $(100 \%)$ représente le domaine d'activité où les répondants s'impliquent le plus ; ensuite vient l'emploi $(97 \%)$; ensuite l'implication dans des comités communautaires (63\%) puis dans des causes sociales comme l'hôpital et les droits des francophones $(57 \%)$ et dans les clubs sportifs $(57 \%$ ). Pour certains interviewés, une seule activité exploite l'entière disponibilité du temps, par exemple, lorsque l'emploi exige d'être disponible vingt-quatre heures par jour et sept jours par semaine, ou lorsque les besoins d'un membre de la famille exigent des soins continus ; pour d'autres, la famille est le lieu central de l'activité. On observe que, dans l'échantillon, six personnes ont une très grande implication dans leur emploi et que cinq en ont une du même ordre dans leur famille. 


\section{Tableau 8 \\ Activités dans lesquelles sont ou ont été impliqués les répondants}

\begin{tabular}{|c|c|c|c|c|c|c|c|c|}
\hline \multirow{2}{*}{$\begin{array}{l}\text { Activités } \\
\text { Famille }\end{array}$} & \multicolumn{2}{|c|}{ Implication } & \multicolumn{2}{|c|}{$\begin{array}{l}\text { Grande } \\
\text { implication }\end{array}$} & \multicolumn{2}{|c|}{$\begin{array}{c}\text { Très grande } \\
\text { implication }\end{array}$} & \multirow{2}{*}{$\begin{array}{l}\mathrm{N} \\
30\end{array}$} & \multirow{2}{*}{$\frac{\%}{100 \%}$} \\
\hline & 14 & $47 \%$ & 11 & $37 \%$ & 5 & $16 \%$ & & \\
\hline Emploi & 14 & $47 \%$ & 9 & $30 \%$ & 6 & $20 \%$ & 29 & $97 \%$ \\
\hline Église & 4 & $13 \%$ & 6 & $20 \%$ & 2 & $7 \%$ & 12 & $40 \%$ \\
\hline $\begin{array}{l}\text { Personnes âgées (Nursing } \\
\text { home, Manoir) }\end{array}$ & 10 & $33 \%$ & 3 & $10 \%$ & 2 & $7 \%$ & 15 & $50 \%$ \\
\hline Municipalité & 9 & $30 \%$ & 2 & $7 \%$ & 0 & $0 \%$ & 11 & $37 \%$ \\
\hline $\begin{array}{l}\text { Organismes (Filles d'Isabelle, } \\
\text { Chevalier de Colomb) }\end{array}$ & 11 & $37 \%$ & 4 & $13 \%$ & 0 & $0 \%$ & 15 & $50 \%$ \\
\hline $\begin{array}{l}\text { Causes (hôpital, auxiliaire, } \\
\text { cause francophone) }\end{array}$ & 8 & $27 \%$ & 6 & $20 \%$ & 3 & $10 \%$ & 17 & $57 \%$ \\
\hline $\begin{array}{l}\text { Club sportif (motoneige, ski } \\
\text { de fond, hockey, volley ball) }\end{array}$ & 12 & $40 \%$ & 4 & $13 \%$ & 1 & $3 \%$ & 17 & $57 \%$ \\
\hline Comités dans la communauté & 13 & $43 \%$ & 4 & $13 \%$ & 2 & $7 \%$ & 19 & $63 \%$ \\
\hline Organismes pour les jeunes & 3 & $10 \%$ & 4 & $13 \%$ & 3 & $10 \%$ & 10 & $33 \%$ \\
\hline $\begin{array}{l}\text { Autres (Société du cancer, } \\
\text { handicapés, défenseurs des } \\
\text { victimes d'agressions } \\
\text { sexuelles) }\end{array}$ & 11 & $37 \%$ & 3 & $10 \%$ & 0 & $0 \%$ & 14 & $47 \%$ \\
\hline
\end{tabular}

Si, en moyenne, les personnes interviewées s'inscrivent dans 6,4 activités, à chacune de ces activités correspond des règles et des obligations qui entrent souvent en contradiction avec une autre de leurs activités (voir tableau 9). Une répondante consacre énormément de temps à son travail ; en même temps, elle est conjointe et mère de famille.

On observe que bon nombre de répondants agissent pour une cause qui les invite à lutter pour d'autres personnes (sens de justice, réaction aux injustices perçues dans les systèmes sociaux, valeurs, croyances). 
Tableau 9

Nombre d'activités dans lesquelles sont impliqués les répondants

\begin{tabular}{lccccccccccc}
\hline Nombre d'activités & 1 & 2 & 3 & 4 & 5 & 6 & 7 & 8 & 9 & 10 & 11 \\
N par nombre d'activités & 0 & 1 & 3 & 2 & 4 & 6 & 4 & 4 & 4 & 2 & 0 \\
$\%$ & 0 & 3 & 10 & 7 & 13 & 20 & 13 & 13 & 13 & 7 & 0 \\
\hline
\end{tabular}

Moyenne de 6,4

\subsection{Défi, passion, amour du métier : des émotions}

Les données indiquent clairement que les intérêts se limitent souvent à la satisfaction, au défi, au plaisir : bref, à une émotion. Comment produire un modèle d'analyse universel utilitaire fondé sur la satisfaction si ces émotions sont différentes pour chacune des relations qu'entretient l'acteur avec ses différents mondes ? Pour un individu donné, les activités familiales fournissent le plaisir et la satisfaction. Pour un autre, les activités communautaires prédominent, sans pour autant qu'il néglige ses activités familiales et son emploi.

Les personnes interrogées parlent des activités qui leur procurent la plus grande émotion. Pour certains, l'emploi est la source de cette émotion ; par exemple, le journaliste, l'entrepreneur ou l'enseignant. Pour d'autres, l'emploi demande beaucoup de temps, les activités qui apportent le plus de satisfaction n'y sont pas directement associées : par exemple, le conseiller municipal, le chef pompier, l'entraineur sportif, le directeur de chorale. Les données indiquent que les acteurs agissent principalement par émotion. L'ensemble des actions s'explique par le plaisir, la satisfaction, le défi, l'amour du métier, l'amour. Que ce soit dans l'emploi ou dans les activités familiales et bénévoles, on peut constater que l'acteur agit par émotion. Mais cette action est aussi rationnelle ; rationnelle, puisque l'acteur n'agit pas de n'importe quelle façon. La rationalité permet de fonctionner, de communiquer, d'interagir, de connaitre les règles et les lois de conduite. L'action, comme ces données le montrent, sans aucun doute possible, est réellement «émorationnelle ».

Plusieurs répondants ont mentionné qu'il était important d'aimer l'activité à laquelle on s'adonnait. Pour le répondant 22 , « il faut que tu aimes ça [l'enseignement], autrement on lâche »; c'est-à-dire que, selon lui, «tu ne peux pas rester dans cette profession que pour le salaire et les belles vacances ". Au contraire, l'intérêt supposerait qu'on aurait des activités auxquelles on n'aimerait pas s'adonner. 


\section{Conclusion}

On aurait pu s'attendre à ce que nos observations montrent que plus l'acteur avance en âge, plus il a tendance à témoigner de rationalité, par exemple, à prendre moins de risques. Toutefois, les données n'indiquent pas de différences à travers les séquences de la vie. On observe que plusieurs des répondantes ont entrepris de grands projets même à un âge avancé, ce qui s'explique en partie par l'émotion qu'occasionnent ces actions. L'explication qui est construite par les personnes interrogées fait état de circonstances, du contexte, des valeurs et du vécu. Pour les jeunes et les moins jeunes, la rationalité est un élément négligeable dans l'explication de l'action. On peut aussi penser que l'instruction laisse présager une plus grande rationalité. Les données ne confirment pas cette présomption. Des répondants détenant un diplôme universitaire orientent leurs actions sur des croyances religieuses et des consultations avec des cartomanciennes. On observe aussi que le sexe et l'état matrimonial n'ont pas de pertinence. Les acteurs sont toujours émorationnels. Même le genre d'activité n'est pas important. Qu'elle soit économique, sociale ou morale, toute action est émorationnelle. L'émotion serait plutôt liée à l'intensité et à la sincérité de l'acteur dans ses actions. Les résultats confirment que la sélection des participants à cette recherche n'a pas en elle-même d'importance.

Cette analyse démontre que l'action humaine est un objet de recherche plus complexe que peut le laisser croire la perspective utilitariste. Toutefois, les modèles de la théorie des choix rationnels continuent de dominer dans l'explication de l'action humaine. Ces modèles considèrent l'action comme «moyen » utilisé par l'acteur pour satisfaire ses intérêts. Pour comprendre l'action, il s'agit de s'interroger sur la rationalité de l'acteur dans l'action. L'acteur calcule tout, l'acteur a l'intention d'entreprendre cette activité, l'acteur est libre. Cette nécessité de préserver la rationalité de l'acteur dans l'action perpétue la compréhension chimérique de l'action. L'analyse des résultats empiriques dans cette recherche met en évidence les limites de cette rationalité de l'acteur. Suite à ces démonstrations, il est nécessaire, pour les sciences sociales, de se dégager des modèles d'analyse de l'action qui ont comme postulat une rationalité exclusive. L'interprétation des données montre bien qu'on abuse de la notion, qu'on déforme la réalité quand on prétend que l'acteur est rationnel, autonome, libre, intentionné et motivé par ses intérêts. Il s'agit là, au mieux, d'éventualité ; on n'a certainement pas affaire à une loi. 
Le paradigme utilitariste a pour postulat une rationalité significative de l'acteur qui permet théoriquement aux spécialistes des sciences sociales de saisir la réalité de l'acteur. Pour soutenir que l'acteur est rationnel, le paradigme suppose qu'il est utilitaire ou, à l'inverse, que si l'acteur est utilitaire, c'est qu'il est rationnel. Cependant, ces modèles négligent des éléments essentiels à leurs explications. L'analyse que nous venons d'effectuer montre bien, d'une part, que la complexité de la réalité de l'action humaine ne peut être réduite à sa simple dimension rationnelle et, d'autre part, que si l'acteur est utilitaire, cela ne se révèle certainement pas dans un calcul avantageux des gains sur les pertes.

En fait, laction résulte d'un ensemble de facteurs, d'éléments, pour l'essentiel inconscients ou imposés à l'acteur, qui, par le produit de cette combinaison, spécifique à une période historique précise, ne consistent pas en un choix, mais en un résultat, dans l'action. L'acteur est un être émorationnel qui, dans chacune de ses relations, agit sur les structures qui le déterminent. Que ce soit un événement important (la mort d'un proche) ou la simple parole d'un ami, tout affecte le dispositif de l'action et reconfigure la structure sociale et les relations. Cette nouvelle combinaison a un effet sur chacune des actions et chacune des relations ultérieures.

De plus, la complexité de la réalité de l'acteur exige une modélisation qui prendrait en considération la multidimensionnalité de l'action. Dans sa vie de tous les jours, l'acteur agit dans différents mondes à la fois. L'acteur n'est pas pour autant cinq ou dix personnes différentes. Il est une seule et même personne agissant dans chacun de ces domaines d'activité. Cependant, tous ces mondes comportent des exigences. 11 est probable que les exigences ne convergent pas toutes dans le même sens : par exemple, le père de famille qui assiste à une conférence et qui, pendant ce temps, ne peut se rendre au spectacle important de son enfant. Comment peut-on présumer que cet homme a évalué toutes ses options et qu'il en est arrivé, après un calcul complexe, à déterminer qu'il subissait moins de pertes en assistant à sa conférence qu'en manquant le spectacle de son enfant?

Comment utiliser la théorie des choix rationnels dans les actions qui impliquent plus d'un acteur? Par exemple : le couple qui achète une entreprise, le couple qui déménage pour l'emploi d'un des membres. Est-ce qu'il s'agit des intérêts de ces deux personnes distinctes ? Certes, il est possible de simplifier en disant que l'emploi apporte le financement à la maison et que chacun en profite, mais qu'est-ce que l'on fait, alors, de toutes les autres dimensions de la vie de chacun des membres de cette famille? Les enfants ont de bons amis et la conjointe aime son emploi et 


\section{PIERRE BOUCHARD / THÉORIE DE L'ACTION ET PARCOURS DE VIE |}

s'implique passionnément au niveau de sa communauté. Au plus, la théorie des choix rationnels fournit une explication normative de telles situations.

Nous avions, au point de départ, un but fondamental qui était de découvrir les limites du paradigme utilitariste. Nous avons aussi voulu vérifier la pertinence de l'usage de concepts fondamentaux du paradigme utilitariste qui servent à légitimer l'idée d'une action ou d'un acteur rationnel. Les concepts de stratégie, d'intention, d'intérêt, d'autonomie se sont avérés inopérants. Après avoir conclu que le modèle utilitariste délaisse des éléments essentiels, nous avons identifié les éléments dont ne peut tenir compte une modélisation utilitariste pour ensuite suggérer un modèle de remplacement qui rassemble l'ensemble des éléments en tenant compte des dynamiques de ce dispositif.

Cette recherche voulait identifier certaines caractéristiques de l'action humaine. Quels sont ces éléments qui font partie intégrante de toute action? Les sciences sociales ne peuvent pas prétendre saisir la réalité de l'acteur si elles ne tiennent pas compte : 1) de la complexité de la réalité de l'acteur ; 2) du fait que l'action soit à la fois émotionnelle et rationnelle ; et 3) de la nécessité de concevoir l'acteur dans la relationalité. Un tel modèle de remplacement serait mieux à même, nous semble-t-il, d'expliquer l'action humaine. 


\section{Références}

Accardo, Alain et Philippe Corcuff, La sociologie de Bourdieu. Textes choisis et commentés, 2 édition revue et augmentée, Bordeaux, Le Mascaret, 1986.

Alexander, Jeffrey C., Theoretical Logic in Sociology, 4 vol., Berkeley, University of California Press, 1982-1983.

Amiot, Michel, Les misères du patronat... Le monde des petites et moyennes entreprises industrielles et de leurs patrons, Paris, L'Harmattan, 1991.

Angers, Maurice, Initiation pratique à la méthodologie des sciences bumaines, Les Éditions CEC, Anjou (Québec), 1996.

Ansart, Pierre, Les sociologies contemporaines, Paris, Seuil, 1990.

Bachelet, Catherine, «GRH et communication interne : quelle place dans les préoccupations des dirigeants de PMI ", Gestion 2000, n" 1, 1995, p. 181-201.

Bauer, Michel, « Patron de PME, âge et rationalité économique », Gérontologie et Société, n" 70, 1994, p. 139-148.

Blais, René, "Perceptions et attitudes à l'égard des ressources humaines ", Relations Industrielles, vol. 49, n' 2, 1994, p. 336-354.

Boltanski, Iuc et Laurent Thévenot, De la justification. Les économies de la grandeur, Paris, Gallimard, essais, 1991.

Boudon, Raymond, «Le "paradoxe du vote" et la théorie de la rationalité », Revue française de sociologie, XXXVIII, 1997, p. 217-227.

Boudon, Raymond, La place du désordre. Critique des théories du changement social, Paris, PUF, 1984.

Boudon, Raymond, La logique du social. Introduction à l'analyse sociologique, Paris, Hachette Littérature, L'esprit critique, 1979.

Boudon, Raymond et François Bourricaud, Dictionnaire critique de la sociologie, Paris, PUF, 1986 [1982].

Bourdieu, Pierre, Raisons Pratiques. Sur la théorie de l'action, Paris, Seuil, 1994.

Buckley, Peter J. et Malcolm Chapman, "Economics and Social Anthropology Reconciling Differences », Human Relations, vol. 49, n" 9, 1996, p. 1123-1149.

Caillé, Alain, Don, intérêt et désintéressement. Bourdieu, Mauss, Platon et quelques auteurs, Paris, La Découverte, 1994.

Caillé, Alain, La démission des clercs, Paris, La Découverte, 1993.

Caillé, Alain, Critique de la raison utilitaire, Paris, La Découverte, 1989.

Caillé, Alain, "La sociologie de l'intérêt est-elle intéressante? ", Sociologie du travail, vol. 23, $\mathrm{n}^{\prime \prime}$ 3, juillet-septembre, 1981, p. 257-274. 


\section{PIERRE BOUCHARD / THÉORIE OELACTION ET PARCOURS DEVE}

Castel, Robert, Les métamorphoses de la question sociale: une chronique du salariat, Paris, Fayard, L'espace du politique, 1995.

Centre de Santé Communautaire Francophone (CSCF), de Kapuskasing et région, Proposal to Establish a Community Health Centre Sering Kapuskasing and Neighbouring Communities of Opasatika, Fauquier, V'al Rita-Harty, and Moonbeam, 1999.

Cova, Bernard, Au-delà du marché. Quand le lien importe plus que le bien, Paris, L'Harmattan, 1995.

Daunais, Jean-Pierre, "L'entretien non directif», in Benoît Gauthier (dir.), Recherche sociale. De la problématique à la collecte des données, Presses de l'Université du Québec, 2 édition, 1992, p. 273-293.

Francfort, Isabelle, Florence Osty, Renaud Sainsaulieu et Marc Uhalde, Les monder socian $\lambda$. de l'entreprise, Paris, Desclée de Brouwer, Sociologie Économique, 1995.

Giroux, Sylvain, Méthodologie des sciences bumaines : la recherche en action, Saint-Laurent (Québec), Éditions du Renouveau Pédagogique, 1998.

Godbout, Jacques T., L'esprit du don, Paris, La Découverte, 1992.

Guerrien, Bernard et Franscico Vergara, "Le MAUSS est un non sens », Revue du MAUSS, $\mathrm{n}^{\circ}$ 6, $2^{\circ}$ semestre, 1995, p. 80-89.

Habermas, Jurgen, « La raison communicationnelle : une autre voie pour sortir de la philosophie du sujet ", Le discours philosophique de la modemite, Paris, Gallimard, Bibliothèque de philosophie, 1987, p. 348-396.

Kets De Vries, Manfred F. R., "The Antomy of the Entrepreneur : Clinical Observations ", Human Relation, vol. 49, n"7, 1996, p. 853-891.

Laflamme, Simon, Humain objet, humain sujet. Initiation à quelques notions de philosophie de l'histoire et d'épistémologie des sciences humaines, Sudbury (Ontario), Université Laurenticnne, Série monographique en sciences humaines, 1996.

Laflamme, Simon, Communication et émotion. Essai de microsociologie relationnelle, Paris, L'Harmattan, 1995.

Laflamme, Simon, La société intégrée. De la circulation des biens, des idées et des personnes, New York/Paris, Peter Lang, Worcester Polytechnic Institute, Studies in Science, Technology and Culture, 1992.

Laflamme, Simon et Rachid Bagaoui, «Don, raison et émotion », Revue de l'Institut de sociologie, vol. 2003, à paraître.

Landry, Réjean, «L'analyse de contenu », in Benoit Gauthier (dir.), Recherche sociale. De la problématique à la collecte des données, Presses de l'Université du Québec, $3^{\circ}$ édition, 1997, p. 329-356.

I.évesque, Benoit, " Repenser l'économie pour contrer l'exclusion sociale : de l'utopie à la nécessité », Cabiers du CRISES, n" 9502, 1997.

Louart, Pierre et Christel Beaucourt, "Pour dynamiser la GRH dans les petites entreprises: dialoguer avec les logiques sociales des dirigeants », Gestion 2000, n" 1, janvier-février, 1995, p. 133-157.

Marin, Bernd, "Qu'est-ce que le patronat? Enjeux théoriques et résultats empiriques ", Sociologie du travail, $\mathrm{n}^{\circ} 4-88,1988$, p. 515-543.

Marseille, Jacques, «Portrait-robot du patron idéal », L'Histoire, n" 189, 1995, p. 6-8.

Mayer, Robert et Marcelle Laforest, «Problème social : le concept et les principales écoles théoriques ", Service Social, vol. 39, n' 2, 1990, p. 13-43.

Morin, Edgar, La méthode, tome 5, L'bumanité de l'bumanité, L'identité bumaine, Paris, Seuil, 2001. 


\section{NPSS, VOLUME 1, NUMÉRO 2, 2006}

Nodier, Luc Marie, "Définition de l'utilitariste », Revue du MAUSS, n" 6, $2^{\mathrm{e}}$ semestre, 1995 , p. $15-30$.

Riboud, Michelle, Accumulation du capital bumain, Paris, Economica, Recherches PanthéonSorbonne, Université de Paris I, Série Sciences Économiques, 1978.

Rocher, Guy, Introduction à la sociologie générale, Montréal, Hurtubise HMH, 3 édition, 1992. Séris, Jean-Pierre, La théorie des jeux, Dossiers Logos dirigés par François Dagognet, Paris, PUF, 1974.

Simon, Herbert Aloys, Science des systemes, sciences de l'artificiel, Paris, Bordas, coll. Afcet Systèmes, 1991 [1969].

Taché, Alain, L'adaptation, un concept sociologique systémique, Paris, L'Harmattan, 2004. 